\title{
Size Classification of Bed Sediment and Selection of Resuspension Monitoring Sites in Upper Tampa Bay, Florida
}

By David H. Schoellhamer

\section{U.S. GEOLOGICAL SURVEY}

Water-Resources Investigations Report 91-4070

Prepared in cooperation with the SOUTHWEST FLORIDA WATER MANAGEMENT DISTRICT, the CITY of TAMPA, the CITY of ST. PETERSBURG, HILLSBOROUGH COUNTY, PINELLAS COUNTY, and the TAMPA PORT AUTHORITY

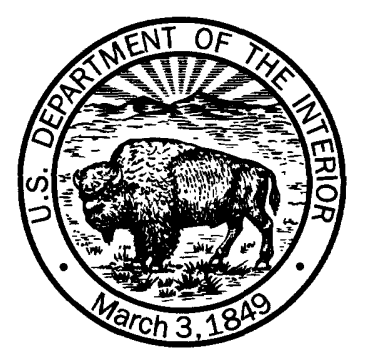




\section{U.S. DEPARTMENT OF THE INTERIOR}

MANUEL LUJAN, JR., Secretary

U.S. GEOLOGICAL SURVEY

Dallas L. Peck, Director

Use of brand names in this report is for identification purposes only and does not constitute endorsement by the U.S. Geological Survey.

For additional information, write to:

\section{District Chief}

U.S. Geological Survey

Suite 3015

227 North Bronough Street

Tallahassee, Florida 32301
Copies of this report may be purchased from:

U.S. Geological Survey

Books and Open-File Reports

Federal Center

Box 25425

Denver, Colorado 80225 


\title{
CONTENTS
}

\author{
Abstract 1 \\ Introduction 1 \\ Purpose and scope 2 \\ Description of study area 2 \\ Previous studies 2 \\ Acknowledgments 4 \\ Data-collection methodology 4 \\ Fathometer transects 4 \\ Grab samples 13 \\ Size classification of bed sediment 16 \\ Selection of resuspension monitoring sites $\mathbf{1 6}$ \\ Summary 22 \\ References 23
}

\section{ILLUSTRATIONS}

Figures 1-4. Maps showing:

1. Location of Tampa Bay study area 3

2. Fathometer transects in middle Tampa Bay $\mathbf{1 0}$

3. Fathometer transects in Hillsborough Bay $\mathbf{1 1}$

4. Fathometer transects in Old Tampa Bay 12

5-6. Graphs showing fathometer output for:

5. Transect 30 in middle Tampa Bay, December 11, 198714

6. Part of transect 10 in western Hillsborough Bay, November 20, 198715

7-9. Maps showing sediment classification at transects in:

7. Middle Tampa Bay, 1987-89 16

8. Hillsborough Bay, 1987-89 17

9. Old Tampa Bay, 1987-89 18

\section{TABLES}

1. Relations among U.S. standard sieve size, microns, phi units, and Wentworth size class $\mathbf{1}$

2. Positions and descriptions of transect nodes in middle Tampa Bay 4

3. Positions and descriptions of transect nodes in Hillsborough Bay east of the ship channel $\mathbf{5}$

4. Positions and descriptions of transect nodes in Hillsborough Bay west of the ship channel $\mathbf{5}$

5. Positions and descriptions of transect nodes in Old Tampa Bay 5

6. Sediment classification interpreted from fathometer transect data for middle Tampa Bay 6

7. Sediment classification interpreted from fathometer transect data for Hillsborough Bay east of the ship channel 7

8. Sediment classification interpreted from fathometer transect data for Hillsborough Bay west of the ship channel 8 
9. Sediment classification interpreted from fathometer transect data for Old Tampa Bay 9

10. Grab sample and interpreted fathometer classifications $\mathbf{1 2}$

11. Results of analyses and classification of sediment grab samples collected from middle Tampa Bay, 1987-89 19

12. Results of analyses and classification of sediment grab samples collected from Hillsborough Bay, 1987-89 20

13. Results of analyses and classification of sediment grab samples collected from Old Tampa Bay, 1987-89 21 
CONVERSION FACTORS, ABBREVIATED WATER-QUALITY UNITS, AND ADDITIONAL ABBREVIATIONS

\begin{tabular}{rcl}
\hline Multiply & By & To obtain \\
\hline inch (in.) & 25.4 & millimeter \\
foot $(\mathrm{ft})$ & 0.3048 & meter \\
yard $(\mathrm{yd})$ & 0.9144 & meter \\
mile $(\mathrm{mi})$ & 1,609 & meter \\
square mile $\left(\mathrm{mi}^{2}\right)$ & 2.590 & square kilometer \\
nautical mile $\left(\mathrm{nmi}^{2}\right)$ & 1,853 & meter \\
$\mathrm{knots}^{2}$ & 0.5144 & meter per second \\
cubic foot per second & 0.02832 & cubic meter per second \\
$(\mathrm{ft} 3 / \mathrm{s})$ & & \\
micron $(\mu \mathrm{m})$ & 0.000001 & meter
\end{tabular}

Abbreviated water-quality unit used in report:

${ }^{\circ} \mathrm{C}=$ degrees Celsius

Additional abbreviations used in report:

$$
\begin{array}{r}
\mathrm{kHz}=\text { kilohertz } \\
\phi=\text { phi units }
\end{array}
$$




\title{
SIZE CLASSIFICATION OF BED SEDIMENT AND SELECTION OF RESUSPENSION MONITORING SITES IN UPPER TAMPA BAY, FLORIDA
}

\author{
By David H. Schoellhamer
}

\begin{abstract}
The size classification of surficial bed sediments in upper Tampa Bay, Florida, including Hillsborough Bay, Old Tampa Bay, and middle Tampa Bay south of the Interbay Peninsula, was determined during 1987-89. Fathometer transects were performed throughout the study area to determine the reflective characteristics of the bed, and grab samples were collected to relate the fathometer data to size classifications of sediments. The data collected during this study indicate that fine bed sediments are most abundant in Hillsborough Bay and least abundant in the southern part of the study area closest to the mouth of Tampa Bay. Generally, the sediments are coarse (mean particle diameter greater than 62.5 microns) in the nearshore shallow water and are finer in the extensive, level, and relatively deep parts of upper Tampa Bay. These size classification data were used to select resuspension monitoring sites in large areas of homogeneous bed sediments in Old Tampa Bay and in Hillsborough Bay.
\end{abstract}

\section{INTRODUCTION}

Estuaries are transition zones between riverine and marine environments. Potential sources of sediment particles for an estuary, such as Tampa Bay, include rivers, net sediment flux from the marine environment, overland runoff, and anthropogenic point sources. Sediment particles are commonly trapped and deposited in the deeper parts of the estuary. The bed sediment affects the overall health of the estuary in several ways. Bed sediments, especially fine sediments, can be resuspended up into the water column where they reduce the amount of light penetrating the water column, can act as a source for constituents adsorbed to the sediment, and can move to undesired locations. The reduction of light in the water column can adversely affect the biological community (Dennison, 1987). Adsorbed constituents that can be released to the water column during suspension, and possibly while on the bed, include nutrients, which can contribute to eutrophication of the estuary, heavy metals, pesticides, and organic carbon compounds that can decrease the productivity of the estuary (Dolan and Bierman, 1982; Li and others, 1984; Grant and Bathmann, 1987; Short, 1987). Resuspended sediment can move throughout the estuary, depending upon the circulation, and can settle in undesired locations, such as shipping channels, turning basins, and marinas (Amos and Tee, 1989; Hamblin, 1989; Lang and others, 1989).

In 1987, the U.S. Geological Survey, in cooperation with the Southwest Florida Water Management District, the city of Tampa, the city of St. Petersburg, Hillsborough County, Pinellas County, and the Tampa Port Authority, began a study titled "Fine sediment resuspension processes and light attenuation in shallow estuarine environments." The objectives of the study are to determine the effect of fine sediment resuspension on light attenuation in Tampa Bay and to determine the mechanisms that cause resuspension of fine sediments. Fine sediments, as used in this report, are defined as sediments with a particle diameter less than $62.5 \mu \mathrm{m}$ or $4.0 \phi$ (phi) units. The relations among U.S. standard sieve size, microns, phi units, and Wentworth size class are given in table 1 . The primary emphasis of the study is to monitor sediment resuspension at selected sites in Tampa Bay. In the initial phase of this study, bottom sediments

Table 1. Relations among U.S. standard sieve size, microns, phi units, and Wentworth size class

\begin{tabular}{|c|c|c|c|}
\hline $\begin{array}{l}\text { U.S. standard } \\
\text { sieve mesh number }\end{array}$ & $\begin{array}{l}\text { Microns } \\
(\mu \mathrm{m})\end{array}$ & $\begin{array}{l}\text { Phi units } \\
(\phi)\end{array}$ & $\begin{array}{l}\text { Wentworth } \\
\text { size class }\end{array}$ \\
\hline 18 & 1,000 & 0.0 & \multirow{3}{*}{ Coarse sand } \\
\hline 25 & 710 & .5 & \\
\hline 35 & 500 & 1.0 & \\
\hline 45 & 350 & 1.5 & \multirow[t]{2}{*}{ Medium sand } \\
\hline 60 & 250 & 2.0 & \\
\hline 80 & 177 & 2.5 & \multirow[t]{2}{*}{ Fine sand } \\
\hline 120 & 125 & 3.0 & \\
\hline 170 & 88 & 3.5 & \multirow[t]{2}{*}{ Very fine sand } \\
\hline 230 & 62.5 & 4.0 & \\
\hline \multirow[t]{14}{*}{325} & 44 & 4.5 & \multirow[t]{2}{*}{ Coarse silt } \\
\hline & 31 & 5.0 & \\
\hline & & & \multirow[t]{2}{*}{ Medium silt } \\
\hline & 15.6 & 6.0 & \\
\hline & & & \multirow[t]{2}{*}{ Fine silt } \\
\hline & 7.8 & 7.0 & \\
\hline & & & \multirow[t]{4}{*}{ Very fine silt } \\
\hline & 3.9 & 8.0 & \\
\hline & 2.0 & 9.0 & \\
\hline & .98 & 10.0 & \\
\hline & .49 & 11.0 & \multirow[t]{4}{*}{ Clay } \\
\hline & .24 & 12.0 & \\
\hline & .12 & 13.0 & \\
\hline & .06 & 14.0 & \\
\hline
\end{tabular}


in the study area were classified according to particle size, and monitoring sites were selected in representative areas.

\section{Purpose and Scope}

The purpose of this report is to describe the particle size classification of the surficial bed sediments in upper Tampa Bay and the selection of resuspension monitoring sites in the area. The most important consideration for site selection is the size of the bed sediment particles. The location and abundance of fine sediments on the bed of upper Tampa Bay are particularly important because they are more susceptible to resuspension. Because deeper sediments are not available for resuspension, only surficial sediments were classified during this study. Determination of sediment stratigraphy is beyond the scope of this study.

Field sampling was limited to parts of Tampa Bay where fine bed sediments were most likely to exist. The study area shown in figure 1 is north of a line from Big Bayou in Pinellas County to about $1.5 \mathrm{mi}$ south of the Little Manatee River in Hillsborough County. South of this line, Goodell and Gorsline (1961) determined that the sediments are predominantly sand that increase in size toward the mouth of the bay. The sediment size in lower Tampa Bay is virtually the same as that described by Goodell and Gorsline. Within the study area, depths less than $3 \mathrm{ft}$ were not sampled because of equipment constraints and because shallowwater sediments are usually sandy because wave energy winnows fine sediments.

\section{Description of Study Area}

Tampa Bay is at the approximate midpoint of the west coast of Florida (fig. 1). The western subembayment of the Y-shaped estuary is Old Tampa Bay, and the eastern subembayment is Hillsborough Bay. The Interbay Peninsula separates the two subembayments. The depth in Tampa Bay generally decreases in a landward direction and the average depth is about $12 \mathrm{ft}$. Hillsborough County encompasses most of the study area except for the western part of Old Tampa Bay and middle Tampa Bay. The city of Tampa is on the eastern shore of Old Tampa Bay and the western and northern shores of Hillsborough Bay. The city of St. Petersburg is at the southwestern boundary of the study area. The surface area of Tampa Bay is about $390 \mathrm{mi}^{2}$, and the surface area of the study area is about $230 \mathrm{mi}^{2}$. A dredged ship channel runs from the mouth of Tampa Bay to about $2 \mathrm{mi}$ south of the Interbay Peninsula where the main channel makes a "T" with the eastern channel proceeding to Tampa and the western channel proceeding to the mouth of Old Tampa Bay.

Total discharge of streams into the study area during water year 1988 was $1,377 \mathrm{ft}^{3} / \mathrm{s}$ (U.S. Geological Survey, 1989). The three largest streams in the study area are the Alafia River (average discharge of $352 \mathrm{ft}^{3} / \mathrm{s}$ during water year 1988) on the eastern shore of Hillsborough Bay, the Hillsborough River $\left(254 \mathrm{ft}^{3} / \mathrm{s}\right)$ on the northern shore of Hillsborough Bay, and the Little Manatee River on the eastern shore of middle Tampa Bay $\left(210 \mathrm{ft}^{3} / \mathrm{s}\right)$. Other sources of inflow include stormwater runoff from the urban commmunities around the bay and treated wastewater outfalls in northern Hillsborough Bay and western Old Tampa Bay. Tampa Bay is a vertically well-mixed estuary because of relatively small inflows and shallow depths. The tides in Tampa Bay are a mixture of both diurnal and semidiurnal components with a range of about $2 \mathrm{ft}$. Average annual water temperatures range from about 21 to $25^{\circ} \mathrm{C}$, and temperature extremes range from about 10 to $32^{\circ} \mathrm{C}$ (Boler, 1987, p. 147).

\section{Previous Studies}

The bed sediments in Tampa Bay are derived from several sources. Originally, terrigenous quartz sand in Pleistocene terrace deposits were eroded and delivered to the bay by streams during the early Holocene (Goodell and Gorsline, 1961). Goodell and Gorsline (1961) state that the Hillsborough River provides a small amount of clay material, but the supply of terrigenous material to the bay is minor because of the low gradients and transport capacities of the major inflowing rivers. These river characteristics have remained unchanged through the present (1990). Littoral drift in the Gulf of Mexico converges at the mouth of Tampa Bay, allowing marine sediments to enter the bay (Willis, 1984). Stahl (1970) stated that the sediments in the bay are primarily of marine origin. Biogenic production of carbonate shell material also is significant in the bay (Goodell and Gorsline, 1961; Doyle, 1975; Willis, 1984).

The mean grain size of surficial bed sediments decreases landward from about 1 to $2 \phi$ units $(250-500 \mu \mathrm{m})$ near the mouth of the bay to 3 to $6 \phi$ units $(15.6-125 \mu \mathrm{m})$ in the northernmost parts of the bay, and most sediment is sand-size (Goodell and Gorsline, 1961; Taylor and Saloman, 1969). The coarsest sediments are found in the deep channels (depth greater than $18 \mathrm{ft}$ ), especially in lower Tampa Bay where the tidal currents are often strongest (Goodell and Gorsline, 1961). Bed sediments with greater than 50 -percent fine material $(4.0 \phi$ units or $62.5 \mu \mathrm{m})$ cover 24 percent of the bottom of Hillsborough Bay (Johansson and Squires, 1989). Isolated areas of Old Tampa Bay contain predominantly fine material (Goodell and Gorsline, 1961; Ross, 1975). Carbonate sediments (shell material) are distributed similarly to the coarser sediments, so carbonates are most abundant in the deep channels of the bay, and their occurrence generally decreases in the landward direction. Sorting generally becomes poorer in the landward direction (Taylor and Saloman, 1969). Poorly sorted sediments, both coarse and fine, generally have the highest content of organic carbon (Goodell and Gorsline, 1961). Comparison of the locations of predominately fine material in Hillsborough Bay (Johansson and Squires, 1989) and the distribution of organic carbon in Hillsborough Bay (U.S. Department of the Interior, 1969) indicate that substantial amounts of fine sediment and organic carbon often exist simultaneously in Hillsborough Bay. This correlation 


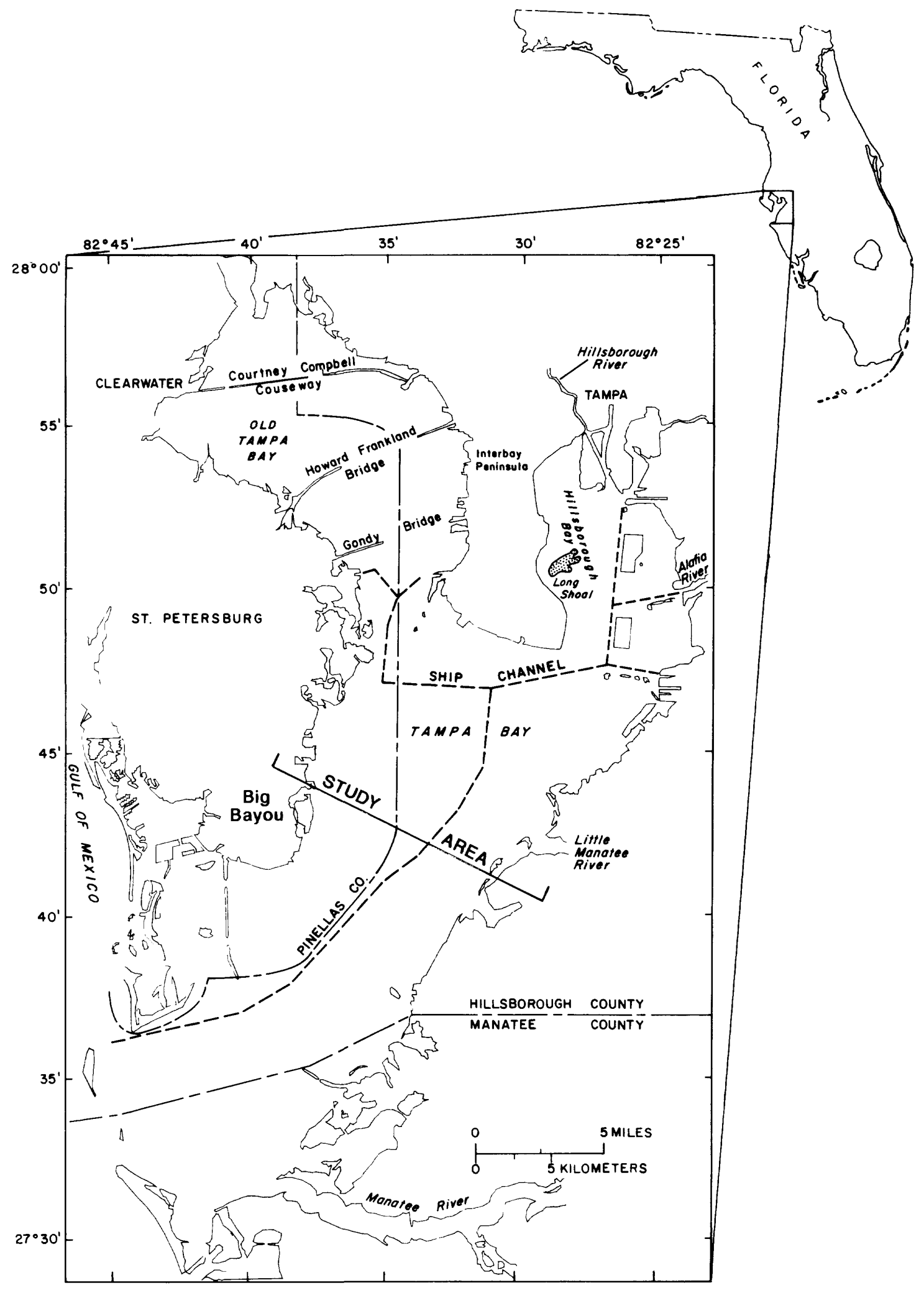

Figure 1. Location of Tampa Bay study area. · 
between fine sediment and organic carbon is probably enhanced by wastewater discharge into Hillsborough Bay.

\section{Acknowledgments}

The data-collection methodology used in this study was previously used in Hillsborough Bay by Roger Johansson and Andy Squires (1989). Grab samples were wet sieved by Robert Hogue and Jonathan Klay of the University of South Florida Geology Department. Pliny Jewell, formerly with the U.S. Geological Survey, provided assistance during this study.

\section{DATA-COLLECTION METHODOLOGY}

The distribution and abundance of fine sediment in upper Tampa Bay were determined with a combination of fathometer transects and grab samples. A fathometer was used to measure the reflectance of sound waves off the bed surface with stronger reflectance indicating hard, less porous, coarse sediments and weaker reflectance indicating soft, more porous, fine sediments. The fathometer has a transducer that transmits sound waves into the water column from near the water surface and receives the signals reflected from the bed. For this study, the transducer was mounted at the stern of a $22-\mathrm{ft}$ vessel to permit rapid measurement over a large area. Grab samples were collected to relate the fathometer data to sediment size classification. The combination of fathometer transects and igrab samples permitted a large area to be measured efficiently and with sufficient accuracy for locating resuspension monitoring sites in large areas of homogeneous bed sediments.

\section{Fathometer Transects}

The transects (or paths) over which the fathometer was operated were determined initially by dividing the study area into four regions: middle Tampa Bay south of the Interbay Peninsula, Hillsborough Bay west of the ship channel, Hillsborough Bay east of the ship channel, and Old Tampa Bay. Areas of fine bottom sediments in Hillsborough Bay were identified in early 1986 (Johansson and Squires, 1989). The study described in this report initially (fall 1987) applied fathometry in Hillsborough Bay in order to familiarize personnel with fathometry techniques in an area with known areas of fine bottom sediments prior to application of fathometry to other areas of Tampa Bay with less wellclassified bottom sediment sizes. The latitude and longitude of navigation aids, bridge openings, piers, and other landmarks were determined from navigation charts to provide starting and stopping points for transects. Transects were selected on the basis of previous studies to include places where fine sediments were expected. Ship channels are discontinuous features of the bay bottom, so they were excluded from most transects.

Knowledge of the position of the vessel performing the transects is necessary for interpretation of the fathometer signal. This study used the Loran-C positioning system that utilizes a special radio receiver to convert the time delays of two Loran-C signals to latitude and longitude. The latitude and longitude were recorded every 2 minutes on the fathometer output. In addition, bearing to the stopping point of the transect and deflection from the desired course were calculated by the Loran- $\mathrm{C}$ unit and monitored during the transects. Comparison of selected recorded coordinates with the desired transects shows that the positioning error was less than about $100 \mathrm{yd}$. Comparison of Loran-C readings with fixed geographical points and navigation aids showed that the Loran-C coordinates were accurate to within about 2 seconds. If a selected starting or stopping point was unreachable due to unexpected shallow water (less than $3 \mathrm{ft}$ ) or if the expected landmark was not present, then the Loran$\mathrm{C}$ coordinates were used to start or stop the transect.

For middle Tampa Bay, Hillsborough Bay east of the ship channel, Hillsborough Bay west of the ship channel, and Old Tampa Bay, tables 2, 3, 4, and 5, respectively, list

Table 2. Positions and descriptions of transect nodes in middle Tampa Bay

[Note: Except where noted, positions and descriptions of landmarks and navigational aids are from the northern Tampa Bay nautical chart (National Oceanic and Atmospheric Administration, 1984)]

\begin{tabular}{|c|c|c|c|}
\hline $\begin{array}{l}\text { Node } \\
\text { number }\end{array}$ & Latitude & Longitude & Description \\
\hline 1 & $27^{\circ} 49^{\prime} 54^{\prime \prime} \mathrm{N}$ & $82^{\circ} 34^{\prime} 48^{\prime \prime} \mathrm{W}$ & End of transect 24 (from Loran-C) \\
\hline 2 & $27^{\circ} 48^{\prime} 33^{\prime \prime} \mathrm{N}$ & $82^{\circ} 26^{\prime} 22^{\prime \prime} \mathrm{W}$ & R "4" Fl R $2.5 \mathrm{sec}$ \\
\hline 3 & $27^{\circ} 47^{\prime} 45^{\prime \prime} \mathrm{N}$ & $82^{\circ} 25^{\prime} 49^{\prime \prime} \mathrm{W}$ & Pile \\
\hline 4 & $27^{\circ} 46^{\prime} 33^{\prime \prime} \mathrm{N}$ & $82^{\circ} 26^{\prime} 2 l^{\prime \prime} \mathrm{W}$ & Fl R 4sec 16ft "2" PA \\
\hline 5 & $27^{\circ} 48^{\prime} 09^{\prime \prime} \mathrm{N}$ & $82^{\circ} 27^{\prime} 54^{\prime \prime} \mathrm{W}$ & R "6" Qk Fl R \\
\hline 6 & $27^{\circ} 44^{\prime} 32^{\prime \prime} \mathrm{N}$ & $82^{\circ} 31^{\prime} 41^{\prime \prime W}$ & R "4E" Fl R 4s \\
\hline 7 & $27^{\circ} 46^{\prime} 43^{\prime \prime} \mathrm{N}$ & $82^{\circ} 31^{\prime} 21^{\prime \prime} \mathrm{W}$ & R "6F" Qk Fl R \\
\hline 8 & $27^{\circ} 43^{\prime} 44^{\prime \prime} \mathrm{N}$ & $82^{\circ} 29^{\prime} 54^{\prime \prime} \mathrm{W}$ & Fl G 4s 16ft 4M "1" \\
\hline 9 & $27^{\circ} 42^{\prime} 37^{\prime \prime} \mathrm{N}$ & $82^{\circ} 31^{\prime} 23^{\prime \prime} \mathrm{W}$ & Iso $\mathrm{R} 6 \mathrm{~s} 59 \mathrm{ft}$ \\
\hline 10 & $27^{\circ} 42^{\prime} 44^{\prime \prime} \mathrm{N}$ & $82^{\circ} 32^{\prime} 21^{\prime \prime} \mathrm{W}$ & $\mathrm{Q} 22 \mathrm{ft}$ \\
\hline 11 & $27^{\circ} 42^{\prime} 45^{\prime \prime} \mathrm{N}$ & $82^{\circ} 33^{\prime} 25^{\prime \prime} \mathrm{W}$ & Iso $6 \mathrm{~s} 59 \mathrm{ft}$ \\
\hline 12 & $27^{\circ} 43^{\prime} 22^{\prime \prime} \mathrm{N}$ & $82^{\circ} 34^{\prime} 18^{\prime \prime} \mathrm{W}$ & Northern marker \\
\hline 13 & $27^{\circ} 44^{\prime} 16^{\prime \prime} \mathrm{N}$ & $82^{\circ} 36^{\prime} 44^{\prime \prime} \mathrm{W}$ & Fl G 2.5s "9" \\
\hline 14 & $27^{\circ} 45^{\prime} 29^{\prime \prime} \mathrm{N}$ & $82^{\circ} 37^{\prime} 35^{\prime \prime} \mathrm{W}$ & $\mathrm{Fl} \mathrm{G} \mathrm{4sec} 16 \mathrm{ft} " 5 "$ \\
\hline 15 & $27^{\circ} 45^{\prime} 33^{\prime \prime} \mathrm{N}$ & $82^{\circ} 37^{\prime} 16^{\prime \prime} \mathrm{W}$ & FI R 4sec 2M "4" \\
\hline 16 & $27^{\circ} 47^{\prime} 40^{\prime \prime} \mathrm{N}$ & $82^{\circ} 35^{\prime} 59^{\prime \prime} \mathrm{W}$ & E Int R $6 \sec 45 \mathrm{ft}$ \\
\hline 17 & $27^{\circ} 48^{\prime} 46^{\prime \prime} \mathrm{N}$ & $82^{\circ} 34^{\prime} 33^{\prime \prime} \mathrm{W}$ & Qk Fl 23ft \\
\hline 21 & $27^{\circ} 47^{\prime} 14^{\prime \prime} \mathrm{N}$ & $82^{\circ} 33^{\prime} 22^{\prime \prime} \mathrm{W}$ & "3G" Fl G 4sec \\
\hline 23 & $27^{\circ} 46^{\prime} 04^{\prime \prime} \mathrm{N}$ & $82^{\circ} 31^{\prime} 26^{\prime \prime} \mathrm{W}$ & C "3F" \\
\hline 26 & $27^{\circ} 51^{\prime} 03^{\prime \prime} \mathrm{N}$ & $82^{\circ} 34^{\prime} 56^{\prime \prime} \mathrm{W}$ & "7" Fl G 4sec \\
\hline 27 & $27^{\circ} 51^{\prime} 32^{\prime \prime} \mathrm{N}$ & $82^{\circ} 35^{\prime} 05^{\prime \prime} \mathrm{W}$ & $\mathrm{R}$ "10" Fl R 4sec \\
\hline 28 & $27^{\circ} 51^{\prime} 24^{\prime \prime} \mathrm{N}$ & $82^{\circ} 34^{\prime} 13^{\prime \prime} \mathrm{W}$ & Pile between cuts on shoal \\
\hline 29 & $27^{\circ} 51^{\prime} 50^{\prime \prime} \mathrm{N}$ & $82^{\circ} 33^{\prime} 16^{\prime \prime} \mathrm{W}$ & C "11K" \\
\hline 30 & $27^{\circ} 49^{\prime} 45^{\prime \prime} \mathrm{N}$ & $82^{\circ} 34^{\prime} 14^{\prime \prime} \mathrm{W}$ & R "2" Fl R 4sec \\
\hline 32 & $27^{\circ} 48^{\prime} 42^{\prime \prime} \mathrm{N}$ & $82^{\circ} 34^{\prime} 08^{\prime \prime} \mathrm{W}$ & G "9" \\
\hline 33 & $27^{\circ} 48^{\prime} 29^{\prime \prime} \mathrm{N}$ & $82^{\circ} 33^{\prime} 09^{\prime \prime} \mathrm{W}$ & R "8" \\
\hline 34 & $27^{\circ} 47^{\prime} 19^{\prime \prime} \mathrm{N}$ & $82^{\circ} 33^{\prime} 19^{\prime \prime} \mathrm{W}$ & $\mathrm{R} N$ "4G" \\
\hline 35 & $27^{\circ} 49^{\prime} 51^{\prime \prime} \mathrm{N}$ & $82^{\circ} 32^{\prime} 22^{\prime \prime} \mathrm{W}$ & Landing lights at end of pier \\
\hline 36 & $27^{\circ} 48^{\prime} 35^{\prime \prime} \mathrm{N}$ & $82^{\circ} 31^{\prime} 49^{\prime \prime} \mathrm{W}$ & $\mathrm{R} " 6$ " \\
\hline 37 & $27^{\circ} 47^{\prime} 12^{\prime \prime} \mathrm{N}$ & $82^{\circ} 32^{\prime} 27^{\prime \prime} \mathrm{W}$ & R "2G" Fl R 4sec BELL \\
\hline 38 & $27^{\circ} 48^{\prime} 38^{\prime \prime} \mathrm{N}$ & $82^{\circ} 30^{\prime} 26^{\prime \prime} \mathrm{W}$ & R "4" \\
\hline 39 & $27^{\circ} 47^{\prime} 29^{\prime \prime} \mathrm{N}$ & $82^{\circ} 30^{\prime} 10^{\prime \prime} \mathrm{W}$ & $\mathrm{Fl} \mathrm{G} 4 \mathrm{sec}$ "1" \\
\hline 40 & $27^{\circ} 48^{\prime} 42^{\prime \prime} \mathrm{N}$ & $82^{\circ} 29^{\prime} 02^{\prime \prime} \mathrm{W}$ & R "2" \\
\hline
\end{tabular}


Table 3. Positions and descriptions of transect nodes in Hillsborough Bay east of the ship channel

[Note: Except where noted, positions and descriptions of landmarks and navigational aids are from the northern Tampa Bay nautical chart (National Oceanic and Atmospheric Administration, 1984)]

\begin{tabular}{|c|c|c|c|}
\hline $\begin{array}{l}\text { Node } \\
\text { number }\end{array}$ & Latitude & Longitude & Description \\
\hline 1 & $27^{\circ} 50^{\prime} 45^{\prime \prime} \mathrm{N}$ & $82^{\circ} 25^{\prime} 28^{\prime \prime} \mathrm{W}$ & End of transect 12 (from Loran-C) \\
\hline 2 & $27^{\circ} 53^{\prime} 49^{\prime \prime} \mathrm{N}$ & $82^{\circ} 26^{\prime} 08^{\prime \prime} \mathrm{W}$ & End of transect 5 (from Loran- C) \\
\hline 3 & $27^{\circ} 50^{\prime} 57^{\prime \prime} \mathrm{N}$ & $82^{\circ} 26^{\prime} 23^{\prime \prime} \mathrm{W}$ & End of transect 4 (from Loran- C) \\
\hline 4 & $27^{\circ} 50^{\prime} 37^{\prime \prime} \mathrm{N}$ & $82^{\circ} 25^{\prime} 28^{\prime \prime} \mathrm{W}$ & End of transect 10 (from Loran-C) \\
\hline 19 & $27^{\circ} 49^{\prime} 11^{\prime \prime} \mathrm{N}$ & $82^{\circ} 26^{\prime} 54^{\prime \prime} \mathrm{W}$ & Qk Fl G "13" \\
\hline 25 & $27^{\circ} 53^{\prime} 04^{\prime \prime} \mathrm{N}$ & $82^{\circ} 26^{\prime} 23^{\prime \prime} \mathrm{W}$ & Fl R 4sec "28" \\
\hline 26 & $27^{\circ} 53^{\prime} 37^{\prime \prime} \mathrm{N}$ & $82^{\circ} 25^{\prime} 57^{\prime \prime} \mathrm{W}$ & $\mathrm{E}$ Int $6 \mathrm{sec} 45 \mathrm{ft}$ \\
\hline 27 & $27^{\circ} 52^{\prime} 50^{\prime \prime} \mathrm{N}$ & $82^{\circ} 25^{\prime} 00^{\prime \prime} \mathrm{W}$ & Northeast node (from Loran-C) \\
\hline 28 & $27^{\circ} 51^{\prime} 01^{\prime \prime} \mathrm{N}$ & $82^{\circ} 25^{\prime} 03^{\prime \prime} \mathrm{W}$ & G "9" \\
\hline 29 & $27^{\circ} 51^{\prime} 44^{\prime \prime} \mathrm{N}$ & $82^{\circ} 26^{\prime} 32^{\prime \prime} \mathrm{W}$ & Fl R 4sec 16ft "24" \\
\hline 30 & $27^{\circ} 50^{\prime} 52^{\prime \prime} \mathrm{N}$ & $82^{\circ} 25^{\prime} 54^{\prime \prime} \mathrm{W}$ & C "5" \\
\hline 31 & $27^{\circ} 50^{\prime} 35^{\prime \prime} \mathrm{N}$ & $82^{\circ} 26^{\prime} 33^{\prime \prime} \mathrm{W}$ & R N "2" \\
\hline 32 & $27^{\circ} 50^{\prime} 53^{\prime \prime} \mathrm{N}$ & $82^{\circ} 25^{\prime} 28^{\prime \prime} \mathrm{W}$ & Fl R 2.5sec $15 \mathrm{ft}$ "8" PA \\
\hline 33 & $27^{\circ} 48^{\prime} 50^{\prime \prime} \mathrm{N}$ & $82^{\circ} 24^{\prime} 50^{\prime \prime} \mathrm{W}$ & Qk Fl R 25ft \\
\hline 34 & $27^{\circ} 49^{\prime} 17^{\prime \prime} \mathrm{N}$ & $82^{\circ} 25^{\prime} 42^{\prime \prime} \mathrm{W}$ & $\mathrm{E}$ Int $6 \mathrm{sec} 59 \mathrm{ft}$ \\
\hline 35 & $27^{\circ} 48^{\prime} 39^{\prime \prime} \mathrm{N}$ & $82^{\circ} 26^{\prime} 46^{\prime \prime} \mathrm{W}$ & "1" Fl G 2.5sec \\
\hline 37 & $27^{\circ} 48^{\prime} 33^{\prime \prime} \mathrm{N}$ & $82^{\circ} 25^{\prime} 52^{\prime \prime} \mathrm{W}$ & "5" Fl 2.5sec \\
\hline
\end{tabular}

Table 4. Positions and descriptions of transect nodes in Hillsborough Bay west of the ship channel

[Note: Except where noted, positions and descriptions of landmarks and navigational aids are from the northern Tampa Bay nautical chart (National Oceanic and Atmospheric Administration, 1984)]

\begin{tabular}{|c|c|c|c|}
\hline $\begin{array}{l}\text { Node } \\
\text { number }\end{array}$ & Latitude & Longitude & Description \\
\hline 1 & $27^{\circ} 51^{\prime} 19^{\prime \prime} \mathrm{N}$ & $82^{\circ} 27^{\prime} 24^{\prime \prime} \mathrm{W}$ & Fl $4 \sec 16 \mathrm{ft} 5 \mathrm{M}$ "1" \\
\hline 3 & $27^{\circ} 54^{\prime} 22^{\prime \prime} \mathrm{N}$ & $82^{\circ} 26^{\prime} 26^{\prime \prime} \mathrm{W}$ & Fl G 2.5sec $16 \mathrm{ft} " 35 "$ \\
\hline 4 & $27^{\circ} 53^{\prime} 20^{\prime \prime} \mathrm{N}$ & $82^{\circ} 28^{\prime} 41^{\prime \prime W}$ & End of Ballast Pt Pier \\
\hline 5 & $27^{\circ} 55^{\prime} 21 " \mathrm{~N}$ & $82^{\circ} 28^{\prime} 03^{\prime \prime} \mathrm{W}$ & Southern pile west of Davis Island \\
\hline 6 & $27^{\circ} 55^{\prime} 39^{\prime \prime} \mathrm{N}$ & $82^{\circ} 27^{\prime} 55^{\prime \prime} \mathrm{W}$ & Northern pile west of Davis Island \\
\hline 7 & $27^{\circ} 55^{\prime} 00^{\prime \prime} \mathrm{N}$ & $82^{\circ} 29^{\prime} 00^{\prime \prime} \mathrm{W}$ & Northwest node (from Loran- C) \\
\hline 8 & $27^{\circ} 53^{\prime} 03^{\prime \prime} \mathrm{N}$ & $82^{\circ} 26^{\prime} 30^{\prime \prime} \mathrm{W}$ & Fl G 6sec "29" \\
\hline 10 & $27^{\circ} 51^{\prime} 36^{\prime \prime N} \mathrm{~N}$ & $82^{\circ} 28^{\prime} 47^{\prime \prime} \mathrm{W}$ & E Int $R 6 \sec 20 \mathrm{ft}$ \\
\hline 11 & $27^{\circ} 52^{\prime} 24^{\prime \prime N}$ & $82^{\circ} 27^{\prime} 31^{\prime \prime W}$ & G "1" \\
\hline 12 & $27^{\circ} 51^{\prime} 40^{\prime \prime} \mathrm{N}$ & $82^{\circ} 26^{\prime} 38^{\prime \prime} \mathrm{W}$ & G "25" Fl 4s \\
\hline 13 & $27^{\circ} 51^{\prime} 33^{\prime \prime} \mathrm{N}$ & $82^{\circ} 28^{\prime} 29^{\prime \prime} \mathrm{W}$ & R "6" \\
\hline 14 & $27^{\circ} 51^{\prime} 58^{\prime \prime} \mathrm{N}$ & $82^{\circ} 28^{\prime} 22^{\prime \prime} \mathrm{W}$ & R "2" \\
\hline 15 & $27^{\circ} 50^{\prime} 40^{\prime \prime} \mathrm{N}$ & $82^{\circ} 26^{\prime} 51^{\prime \prime} \mathrm{W}$ & Qk Fl R 15ft \\
\hline 16 & $27^{\circ} 50^{\prime} 45^{\prime \prime} \mathrm{N}$ & $82^{\circ} 26^{\prime} 43^{\prime \prime} \mathrm{W}$ & Fl G 4sec "21" \\
\hline 17 & $27^{\circ} 51^{\prime} 28^{\prime \prime} \mathrm{N}$ & $82^{\circ} 28^{\prime} 42^{\prime \prime} \mathrm{W}$ & G "9" \\
\hline 18 & $27^{\circ} 50^{\prime} 29^{\prime \prime} \mathrm{N}$ & $82^{\circ} 27^{\prime} 48 \mathrm{~W}$ & E Int $R 6 s$ 46ft \\
\hline 19 & $27^{\circ} 49^{\prime} 11 " \mathrm{~N}$ & $82^{\circ} 26^{\prime} 54^{\prime \prime} \mathrm{W}$ & Qk Fl G "13" \\
\hline 20 & $27^{\circ} 48^{\prime} 41^{\prime \prime N}$ & $82^{\circ} 27^{\prime} 31^{\prime \prime W}$ & Qk Fl R 25ft \\
\hline 22 & $27^{\circ} 48^{\prime} 54^{\prime \prime N}$ & $82^{\circ} 27^{\prime} 01^{\prime \prime W}$ & Fl G $2.5 \sec " 11 "$ \\
\hline 23 & $27^{\circ} 50^{\prime} 17^{\prime \prime} \mathrm{N}$ & $82^{\circ} 26^{\prime} 47^{\prime \prime} \mathrm{W}$ & C"19" \\
\hline 24 & $27^{\circ} 53^{\prime} 50^{\prime \prime} \mathrm{N}$ & $82^{\circ} 26^{\prime} 39^{\prime \prime} \mathrm{W}$ & E Int $G 6 \sec 35 \mathrm{ft}$ \\
\hline 39 & $27^{\circ} 48^{\prime} 48^{\prime \prime N}$ & $82^{\circ} 28^{\prime} 48^{\prime \prime} \mathrm{W}$ & E Int R 4sec 50ft \\
\hline 40 & $27^{\circ} 54^{\prime} 13^{\prime \prime} \mathrm{N}$ & $82^{\circ} 27^{\prime} 03^{\prime \prime} \mathrm{W}$ & End of transect 4 (from Loran- C) \\
\hline 41 & $27^{\circ} 52^{\prime} 15^{\prime \prime N}$ & $82^{\circ} 29^{\prime} 03^{\prime \prime} \mathrm{W}$ & End of transect 20 (from Loran- C) \\
\hline
\end{tabular}

Table 5. Positions and descriptions of transect nodes in Old Tampa Bay

[Note: Except where noted, positions and descriptions of landmarks and navigational aids are from the northern Tampa Bay nautical chart (National Oceanic and Atmospheric Administration, 1984)]

\begin{tabular}{|c|c|c|c|}
\hline $\begin{array}{l}\text { Node } \\
\text { number }\end{array}$ & Latitude & Longitude & Description \\
\hline 4 & $27^{\circ} 59^{\prime} 16^{\prime \prime} \mathrm{N}$ & $82^{\circ} 41^{\prime} 06^{\prime \prime} \mathrm{W}$ & Dock north of Cooper Point \\
\hline 5 & $27^{\circ} 59^{\prime} 12^{\prime \prime} \mathrm{N}$ & $82^{\circ} 37^{\prime} 25^{\prime \prime} \mathrm{W}$ & Cabbage Bayou "R2" \\
\hline 6 & $27^{\circ} 57^{\prime} 46^{\prime \prime N} \mathrm{~N}$ & $82^{\circ} 40^{\prime} 41^{\prime \prime W}$ & $\begin{array}{l}\text { Center of western Courtney } \\
\text { Cambell Bridge }\end{array}$ \\
\hline 7 & $27^{\circ} 58^{\prime} 11 " \mathrm{~N}$ & $82^{\circ} 37^{\prime} 30^{\prime \prime} \mathrm{W}$ & $\begin{array}{l}\text { Center of eastern Courtney } \\
\text { Cambell Bridge }\end{array}$ \\
\hline 8 & $27^{\circ} 56^{\prime} 30^{\prime \prime} \mathrm{N}$ & $82^{\circ} 43^{\prime} 02^{\prime \prime} \mathrm{W}$ & $\begin{array}{l}\text { Easternmost Largo Inlet } \\
\text { channel marker }\end{array}$ \\
\hline 10 & $27^{\circ} 55^{\prime} 56^{\prime \prime} \mathrm{N}$ & $82^{\circ} 41^{\prime} 32^{\prime \prime} \mathrm{W}$ & Point north of St. Petersburg Airpor \\
\hline 11 & $27^{\circ} 55^{\prime} 11^{\prime \prime} \mathrm{N}$ & $82^{\circ} 36^{\prime} 34^{\prime \prime W}$ & $\begin{array}{l}\text { Western end of the Howard } \\
\text { Frankland Bridge }\end{array}$ \\
\hline 12 & $27^{\circ} 55^{\prime} 39^{\prime \prime} \mathrm{N}$ & $82^{\circ} 35^{\prime} 14^{\prime \prime} \mathrm{W}$ & Center of Howard Frankland Bridge \\
\hline 13 & $27^{\circ} 56^{\prime} 08^{\prime \prime} \mathrm{N}$ & $82^{\circ} 33^{\prime} 52^{\prime \prime} \mathrm{W}$ & $\begin{array}{l}\text { Eastern end of the Howard } \\
\text { Frankland Bridge }\end{array}$ \\
\hline 14 & $27^{\circ} 56^{\prime} 06^{\prime \prime} \mathrm{N}$ & $82^{\circ} 32^{\prime} 59^{\prime \prime} \mathrm{W}$ & Culbreath Bayou "R2" \\
\hline 15 & $27^{\circ} 55^{\prime} 05^{\prime \prime} \mathrm{N}$ & $82^{\circ} 32^{\prime} 19^{\prime \prime} \mathrm{W}$ & Marker west of John Brook \\
\hline 17 & $27^{\circ} 52^{\prime} 43^{\prime \prime} \mathrm{N}$ & $82^{\circ} 34^{\prime} 58^{\prime \prime W}$ & Western end of the Gandy Bridge \\
\hline 18 & $27^{\circ} 53^{\prime} 15^{\prime \prime} \mathrm{N}$ & $82^{\circ} 33^{\prime} 06^{\prime \prime} \mathrm{W}$ & Pass under Gandy Bridge \\
\hline 19 & $27^{\circ} 52^{\prime} 38^{\prime \prime} \mathrm{N}$ & $82^{\circ} 34^{\prime} 57^{\prime \prime} \mathrm{W}$ & South Gandy Channel "G1" \\
\hline 21 & $27^{\circ} 51^{\prime} 43^{\prime \prime} \mathrm{N}$ & $82^{\circ} 35^{\prime} 12^{\prime \prime} \mathrm{W}$ & Qk Fl R 25ft \\
\hline 22 & $27^{\circ} 52^{\prime} 13^{\prime \prime} \mathrm{N}$ & $82^{\circ} 33^{\prime} 23^{\prime \prime} \mathrm{W}$ & Qk Fl G $14 \mathrm{ft}$ \\
\hline 23 & $27^{\circ} 52^{\prime} 01^{\prime \prime} \mathrm{N}$ & $82^{\circ} 32^{\prime} 52^{\prime \prime} \mathrm{W}$ & Qk Fl R $14 \mathrm{ft}$ \\
\hline 24 & $27^{\circ} 57^{\prime} 42^{\prime \prime} \mathrm{N}$ & $82^{\circ} 34^{\prime} 32^{\prime \prime} \mathrm{W}$ & End of transect 9 (from Loran- $C$ ) \\
\hline 25 & $27^{\circ} 57^{\prime} 32 " \mathrm{~N}$ & $82^{\circ} 34^{\prime} 22^{\prime \prime} \mathrm{W}$ & Start of transect 13 (from Loran-C) \\
\hline 27 & $27^{\circ} 52^{\prime} 45^{\prime \prime} \mathrm{N}$ & $82^{\circ} 35^{\prime} 59^{\prime \prime} \mathrm{W}$ & End of transect 19 (from Loran- C) \\
\hline 28 & $27^{\circ} 57^{\prime} 39^{\prime \prime} \mathrm{N}$ & $82^{\circ} 34^{\prime} 30^{\prime \prime} \mathrm{W}$ & Start of transect 14 (from Loran-C) \\
\hline 29 & $27^{\circ} 54^{\prime} 16^{\prime \prime} \mathrm{N}$ & $82^{\circ} 37^{\prime} 22^{\prime \prime} \mathrm{W}$ & End of transect 17 (from Loran- C) \\
\hline
\end{tabular}

the number, position, and description of the transect nodes, and tables $6,7,8$, and 9 give the numbers of the nodes used to start and stop each transect. Figures 2, 3, and 4 are maps of the transects in middle Tampa Bay, Hillsborough Bay, and Old Tampa Bay, respectively. For example, from tables 5 and 9 and figure 4, Old Tampa Bay transect 12 extends from node 12, the center of the Howard Frankland Bridge $\left(27^{\circ} 55^{\prime} 39^{\prime \prime} \mathrm{N}, 82^{\circ} 35^{\prime} 14^{\prime \prime} \mathrm{W}\right)$, to node 7 , the center of the eastern Courtney Campbell Causeway bridge $\left(27^{\circ} 58^{\prime} 11^{\prime \prime} \mathrm{N}\right.$, $\left.82^{\circ} 37^{\prime} 30^{\prime \prime} \mathrm{W}\right)$. Node and transect numbering is discontinuous in each area because some numbers were assigned but not used. Most of the landmarks used are included on the navigation chart for northern Tampa Bay (National Oceanic and Atmospheric Administration, 1984).

The fathometer used was a Raytheon model DE-719C, which operates at $208 \mathrm{kHz}$ (Raytheon Marine Company, 1982). The transducer was mounted at the stern of a $22-\mathrm{ft}$ vessel and the output was recorded on a strip chart. When a fathometer is used to measure the depth of water, the 
Table 6. Sediment classification interpreted from fathometer transect data for middle Tampa Bay

[Note: The location along the transect is expressed as a percentage of the distance from the starting node to the stopping node of the transect; --, no data]

\begin{tabular}{|c|c|c|c|c|c|c|}
\hline $\begin{array}{l}\text { Transect } \\
\text { number }\end{array}$ & $\begin{array}{l}\text { From } \\
\text { node }\end{array}$ & $\begin{array}{c}\text { To } \\
\text { node }\end{array}$ & $\begin{array}{l}\text { Coarse } \\
\text { material } \\
\text { (percent) }\end{array}$ & $\begin{array}{c}\text { Coarse } \\
\text { with fines } \\
\text { (percent) }\end{array}$ & $\begin{array}{c}\text { Fine } \\
\text { material } \\
\text { (percent) }\end{array}$ & $\begin{array}{c}\text { Spoil } \\
\text { (percent) }\end{array}$ \\
\hline 1 & 2 & 3 & $0-25$ & $25-100$ & -- & -- \\
\hline 2 & 3 & 4 & $59-100$ & $0-18$ & -- & $18-59$ \\
\hline 3 & 4 & 5 & $0-39$ & $39-59$ & $59-80$ & $80-100$ \\
\hline \multirow[t]{3}{*}{4} & 5 & 6 & $48-63$ & $18-48$ & -- & $6-18$ \\
\hline & & & $67-77$ & -- & -- & $63-67$ \\
\hline & & & -- & -- & - & $77-100$ \\
\hline \multirow[t]{2}{*}{5} & 6 & 4 & $10-17$ & -- & -- & $0-10$ \\
\hline & & & $21-100$ & -- & -- & $17-21$ \\
\hline \multirow[t]{2}{*}{6} & 4 & 7 & $0-60$ & $60-66$ & -- & $66-81$ \\
\hline & & & -- & $81-86$ & -- & $86-100$ \\
\hline 7 & 8 & 7 & $0-100$ & -- & - & -- \\
\hline 8 & 9 & 8 & $0-100$ & -- & -- & -- \\
\hline 9 & 10 & 9 & $60-100$ & -- & -- & $0-60$ \\
\hline 11 & 11 & 10 & $0-24$ & -- & -- & $24-100$ \\
\hline 12 & 12 & 11 & $0-100$ & -. & -- & -- \\
\hline 13 & 13 & 12 & $0-100$ & -- & -- & -- \\
\hline 14 & 14 & 13 & $0-100$ & -- & -- & -- \\
\hline 15 & 16 & 15 & $0-100$ & -- & - & -- \\
\hline 16 & 17 & 16 & $0-100$ & -- & - & -- \\
\hline 17 & 16 & 12 & $0-100$ & - & -- & - \\
\hline 21 & 12 & 21 & $0-83$ & $83-100$ & -- & -- \\
\hline \multirow[t]{4}{*}{23} & 23 & 16 & $0-5$ & $8-23$ & -- & $5-8$ \\
\hline & & & $23-37$ & $37-57$ & -- & -- \\
\hline & & & $57-74$ & $74-86$ & -. & -- \\
\hline & & & $86-100$ & -- & -- & -. \\
\hline \multirow[t]{2}{*}{24} & 26 & 1 & $0-19$ & - & -- & $19-64$ \\
\hline & & & $64-100$ & -- & -- & -- \\
\hline 25 & 28 & 27 & $0-100$ & -- & -- & -- \\
\hline 26 & 28 & 29 & $0-92$ & -- & -- & $92-100$ \\
\hline 27 & 30 & 28 & $0-100$ & -- & -- & -- \\
\hline 29 & 32 & 33 & $0-100$ & -- & -- & -- \\
\hline \multirow[t]{2}{*}{30} & 33 & 34 & $0-68$ & -- & $83-98$ & $68-83$ \\
\hline & & & -- & -- & -- & $98-100$ \\
\hline 31 & 33 & 35 & $0-100$ & -- & - & -- \\
\hline 32 & 36 & 33 & $0-100$ & -- & -- & -- \\
\hline \multirow[t]{2}{*}{33} & 37 & 36 & $33-77$ & $77-98$ & $0-33$ & -- \\
\hline & & & $98-100$ & -- & -- & -- \\
\hline 34 & 38 & 35 & $0-100$ & -- & -- & -- \\
\hline 35 & 36 & 38 & $0-100$ & -- & -- & -- \\
\hline 36 & 39 & 36 & $17-100$ & -- & $0-17$ & -- \\
\hline 38 & 40 & 38 & $0-100$ & -- & -- & -- \\
\hline 39 & 38 & 39 & $0-44$ & -- & $78-100$ & $44-78$ \\
\hline 40 & 39 & 40 & $47-100$ & -- & $0-33$ & $33-47$ \\
\hline
\end{tabular}


[Note: The location along the transect is expressed as a percentage of the distance from the starting node to the stopping node of the transect; --, no data]

\begin{tabular}{|c|c|c|c|c|c|c|}
\hline $\begin{array}{l}\text { Transect } \\
\text { number }\end{array}$ & $\begin{array}{l}\text { From } \\
\text { node }\end{array}$ & $\begin{array}{c}\text { To } \\
\text { node }\end{array}$ & $\begin{array}{l}\text { Coarse } \\
\text { material } \\
\text { (percent) }\end{array}$ & $\begin{array}{c}\text { Coarse } \\
\text { with fines } \\
\text { (percent) }\end{array}$ & $\begin{array}{c}\text { Fine } \\
\text { material } \\
\text { (percent) }\end{array}$ & $\begin{array}{c}\text { Spoil } \\
\text { (percent) }\end{array}$ \\
\hline \multirow[t]{2}{*}{1} & 26 & 27 & $0-5$ & -- & $5-10$ & -- \\
\hline & & & $10-100$ & -- & -- & -- \\
\hline \multirow[t]{2}{*}{2} & 27 & 28 & $0-12$ & -- & $12-59$ & -- \\
\hline & & & $59-100$ & -- & -- & -- \\
\hline 3 & 28 & 29 & 0-29 & -- & $29-85$ & $85-100$ \\
\hline \multirow[t]{3}{*}{4} & 25 & 3 & -- & -- & $13-64$ & $0-13$ \\
\hline & & & -- & -- & $88-94$ & $64-88$ \\
\hline & & & -- & -- & -- & $94-100$ \\
\hline 5 & 25 & 2 & $95-100$ & -- & $47-95$ & $0-47$ \\
\hline \multirow[t]{2}{*}{6} & 25 & 26 & $0-46$ & -- & $46-96$ & - \\
\hline & & & $96-100$ & - & -- & -- \\
\hline \multirow[t]{2}{*}{7} & 30 & 27 & $25-50$ & -- & $0-25$ & -- \\
\hline & & & $85-100$ & -- & $50-85$ & - \\
\hline \multirow[t]{4}{*}{8} & 31 & 35 & $8-19$ & $80-93$ & -- & $0-8$ \\
\hline & & & $25-46$ & -- & -- & $19-25$ \\
\hline & & & -- & -- & -- & $46-80$ \\
\hline & & & -- & -- & -- & $93-100$ \\
\hline \multirow[t]{2}{*}{9} & 35 & 33 & $92-100$ & $15-45$ & -- & $0-15$ \\
\hline & & & -- & -- & -- & $45-92$ \\
\hline 10 & 33 & 4 & $0-79$ & -- & -- & $79-100$ \\
\hline 11 & 31 & 32 & $88-100$ & - & $22-88$ & $0-22$ \\
\hline \multirow[t]{2}{*}{12} & 34 & 1 & $0-72$ & -- & $72-93$ & -- \\
\hline & & & $93-100$ & - & -- & - \\
\hline 14 & 35 & 34 & $73-100$ & - & $41-73$ & $0-41$ \\
\hline 15 & 34 & 19 & $0-10$ & -- & -- & $10-100$ \\
\hline 16 & 34 & 33 & $0-100$ & -- & -- & -- \\
\hline \multirow[t]{2}{*}{17} & 34 & 37 & $0-4$ & -- & -- & $4-71$ \\
\hline & & & $71-100$ & - & -- & -- \\
\hline
\end{tabular}

sensitivity is set so that only one signal is received from the bottom. This signal begins at the transducer, propagates to the bottom at the speed of sound, reflects off the bottom, is received by the transducer, and is recorded on the strip chart. The time delay is related to the distance the signal traveled, so the position of the line on the strip chart indicates the depth of water. The strength of the signal received is diminished by absorption at the bottom and attenuation in the water column. After passing the transducer, this signal reflects off the water surface and again propagates to the bottom, reflects off the bottom, and is received by the transducer again. When the sensitivity of the fathometer is set high enough, this second signal is recorded on the strip chart. Because the second signal reflects off the bottom sediments twice, its strength is more dependent on the type of bottom sediment than the first signal. Hard, shelly, or sandy sediments are less porous and more reflective than softer, more porous, finer sediments (Smith and Li, 1966; Hamilton, 1970). The reflected signals, and preferably the second signal, can be used to identify the type of bottom material when interpreted in conjunction with grab samples (Smith and Li, 1966; Raytheon Marine Company, 1982; Johansson and Squires, 1989). Under some conditions, a fathometer can be used to determine the thickness of fine bottom sediment (Kirby and others, 1989), but this type of analysis was beyond the scope of this study. 
Table 8. Sediment classification interpreted from fathometer transect data for Hillsborough Bay west of the ship channel

[Note: The location along the transect is expressed as a percentage of the distance from the starting node to the stopping node of the transect; --, no data]

\begin{tabular}{|c|c|c|c|c|c|c|}
\hline $\begin{array}{l}\text { Transect } \\
\text { number }\end{array}$ & $\begin{array}{l}\text { From } \\
\text { node }\end{array}$ & $\begin{array}{c}\text { To } \\
\text { node }\end{array}$ & $\begin{array}{l}\text { Coarse } \\
\text { material } \\
\text { (percent) }\end{array}$ & $\begin{array}{c}\text { Coarse } \\
\text { with fines } \\
\text { (percent) }\end{array}$ & $\begin{array}{c}\text { Fine } \\
\text { material } \\
\text { (percent) }\end{array}$ & $\begin{array}{c}\text { Spoil } \\
\text { (percent) }\end{array}$ \\
\hline 1 & 20 & 39 & $75-100$ & -- & -- & $0-75$ \\
\hline 2 & 18 & 20 & $0-18$ & .. & $18-31$ & $31-100$ \\
\hline 3 & 1 & 18 & $36-100$ & -- & $0-36$ & - \\
\hline \multirow[t]{3}{*}{4} & 1 & 40 & $26-34$ & $63-67$ & $0-26$ & -- \\
\hline & & & $67-81$ & $97-100$ & $34-63$ & -- \\
\hline & & & -- & -- & $81-97$ & -- \\
\hline \multirow[t]{2}{*}{5} & 3 & 4 & $0-9$ & $82-86$ & $9-32$ & -- \\
\hline & & & $32-82$ & $95-100$ & $86-95$ & - \\
\hline \multirow[t]{2}{*}{6} & 4 & 5 & $26-68$ & $0-14$ & $14-26$ & -- \\
\hline & & & -. & -. & $68-100$ & -- \\
\hline \multirow[t]{2}{*}{7} & 5 & 6 & $0-36$ & .. & $36-59$ & -- \\
\hline & & & $59-100$ & -- & -- & -- \\
\hline 9 & 5 & 7 & $0-16$ & $16-100$ & -- & -- \\
\hline \multirow[t]{2}{*}{10} & 7 & 4 & - & $0-25$ & $25-84$ & -- \\
\hline & & & - & $84-100$ & - & -- \\
\hline 11 & 24 & 7 & $20-72$ & $72-100$ & $0-20$ & - \\
\hline \multirow[t]{2}{*}{14} & 4 & 8 & $95-100$ & $0-4$ & $4-22$ & -- \\
\hline & & & - & $22-95$ & -- & -- \\
\hline \multirow[t]{2}{*}{15} & 4 & 11 & $80-100$ & $0-38$ & $38-73$ & -- \\
\hline & & & -- & $73-80$ & -- & -- \\
\hline \multirow[t]{2}{*}{17} & 11 & 12 & $0-28$ & -- & $28-94$ & -. \\
\hline & & & $94-100$ & -- & -- & -- \\
\hline \multirow[t]{2}{*}{19} & 10 & 4 & $0-8$ & 98-100 & $8-59$ & -- \\
\hline & & & $59-98$ & -- & -- & - \\
\hline \multirow[t]{2}{*}{20} & 8 & 41 & $0-5$ & $5-20$ & $20-41$ & -- \\
\hline & & & $94-100$ & $41-59$ & $59-94$ & -- \\
\hline \multirow[t]{2}{*}{21} & 13 & 8 & $28-59$ & $90-100$ & $0-28$ & -- \\
\hline & & & - & -- & $59-90$ & -- \\
\hline 22 & 12 & 13 & $0-7$ & -- & $7-100$ & -- \\
\hline 24 & 13 & 14 & $84-100$ & -- & $0-84$ & - \\
\hline 26 & 17 & 1 & $0-9$ & $9-89$ & $89-100$ & -- \\
\hline 27 & 16 & 17 & $43-100$ & -. & $0-43$ & - \\
\hline 30 & 20 & 22 & - & -. & -- & $0-100$ \\
\hline \multirow[t]{2}{*}{33} & 12 & 24 & $0-12$ & $12-18$ & $18-38$ & -- \\
\hline & & & - & $38-76$ & $76-100$ & -- \\
\hline 34 & 12 & 15 & - & - & $0-100$ & -- \\
\hline \multirow[t]{2}{*}{35} & 15 & 19 & - & $26-55$ & $0-2$ & $2-26$ \\
\hline & & & $\ldots$ & -- & - & $55-100$ \\
\hline \multirow[t]{2}{*}{36} & 18 & 15 & $0-4$ & -- & $4-79$ & $79-92$ \\
\hline & & & -- & - & $92-100$ & -- \\
\hline 37 & 23 & 18 & $95-100$ & -- & $47-95$ & $0-47$ \\
\hline 38 & 20 & 15 & - & - & -- & $0-100$ \\
\hline 39 & 19 & 18 & $97-100$ & -- & $47-97$ & $0-47$ \\
\hline
\end{tabular}


Table 9. Sediment classification interpreted from fathometer transect data for Old Tampa Bay

[Note: The location along the transect is expressed as a percentage of the distance from the starting node to the stopping node of the transect; --, no data]

\begin{tabular}{|c|c|c|c|c|c|c|}
\hline $\begin{array}{l}\text { Transect } \\
\text { number }\end{array}$ & $\begin{array}{l}\text { From } \\
\text { node }\end{array}$ & $\begin{array}{c}\text { To } \\
\text { node }\end{array}$ & $\begin{array}{l}\text { Coarse } \\
\text { material } \\
\text { (percent) }\end{array}$ & $\begin{array}{c}\text { Coarse } \\
\text { with fines } \\
\text { (percent) }\end{array}$ & $\begin{array}{c}\text { Fine } \\
\text { material } \\
\text { (percent) }\end{array}$ & $\begin{array}{c}\text { Spoil } \\
\text { (percent) }\end{array}$ \\
\hline \multirow[t]{2}{*}{3} & 4 & 5 & $0-18$ & $18-25$ & -- & - \\
\hline & & & $25-100$ & -- & -- & -- \\
\hline \multirow[t]{3}{*}{4.} & 7 & 4 & $0-43$ & $43-54$ & -- & -- \\
\hline & & & $54-65$ & $65-83$ & -- & -- \\
\hline & & & $83-100$ & -- & -- & -- \\
\hline \multirow[t]{2}{*}{5} & 5 & 6 & $0-51$ & $51-81$ & $81-97$ & -- \\
\hline & & & $97-100$ & -- & -- & -- \\
\hline \multirow[t]{2}{*}{7} & 8 & 7 & $0-1$ & $59-80$ & $1-23$ & $80-84$ \\
\hline & & & $23-59$ & $84-93$ & -- & $93-100$ \\
\hline 9 & 7 & 24 & $0-100$ & -- & -- & -- \\
\hline \multirow[t]{2}{*}{10} & 6 & 10 & $0-65$ & -- & $65-98$ & -- \\
\hline & & & $98-100$ & -- & -- & -- \\
\hline 11 & 11 & 6 & $0-100$ & -- & -- & -- \\
\hline \multirow[t]{3}{*}{12} & 12 & 7 & $0-1$ & $1-13$ & -- & -- \\
\hline & & & $13-23$ & $23-71$ & -- & -- \\
\hline & & & $71-100$ & -- & -- & -- \\
\hline 13 & 25 & 13 & $0-100$ & -- & -- & -- \\
\hline \multirow[t]{3}{*}{14} & 28 & 10 & $0-2$ & $2-13$ & -- & -- \\
\hline & & & $13-18$ & $18-54$ & -- & -- \\
\hline & & & $54-85$ & $85-100$ & -- & -- \\
\hline 15 & 10 & 8 & $0-100$ & -- & -- & -- \\
\hline 16 & 10 & 12 & $2-100$ & $0-2$ & -- & -- \\
\hline 17 & 12 & 29 & $0-100$ & -- & -- & -- \\
\hline \multirow[t]{2}{*}{19} & 11 & 27 & $0-3$ & -- & -- & $3-5$ \\
\hline & & & $5-100$ & -- & -- & -- \\
\hline 21 & 17 & 14 & $0-100$ & -- & -- & -- \\
\hline 22 & 15 & 14 & $44-100$ & -- & -- & $0-44$ \\
\hline 23 & 18 & 15 & $0-99$ & -- & -- & $99-100$ \\
\hline 24 & 19 & 18 & $0-100$ & -- & -- & -- \\
\hline 25 & 18 & 23 & $0-100$ & -- & -- & -- \\
\hline 26 & 23 & 22 & $0-100$ & -- & -- & -- \\
\hline 27 & 22 & 21 & $0-100$ & -- & -- & -- \\
\hline 28 & 21 & 19 & $0-100$ & -- & -- & -- \\
\hline
\end{tabular}

There are several difficulties and limitations to interpreting fathometer signals. In the studies of Tampa Bay, the fathometer signal generally penetrated the bottom sediments only a short distance so interpretation of sediment type from fathometer data applies only to the surficial sediments. Water attenuates the fathometer signal so an increase in depth decreases the strength of the received signal; therefore, during this study, fathometer sensitivity was adjusted for significant changes in depth, and these adjustments were marked on the chart so they could be properly interpreted. The strength of the signal also would decrease if the vessel was moving at greater than 10 knots, so a constant speed in the range of 6 to 10 knots was used for each transect.
The fathometer transect data, in conjunction with grab samples, were used to classify the bottom sediment as either coarse, coarse with fines, fine, or spoil. The criteria used for the classification of particle size are presented in table 10 . Coarse sediments are defined as sediments with less than 12 percent by weight passing a $62.5-\mu \mathrm{m}$ sieve, and coarse sediments with fine material are defined as having between 12 and 50 percent by weight passing a $62.5-\mu \mathrm{m}$ sieve (Bowles, 1978). For purposes of interpreting the fathometer data, all sediments with more than 50 percent by weight passing a $62.5-\mu \mathrm{m}$ sieve were classified as fine. In areas of irregular bottom topography due to placement of dredged material, the sediment size classification could not be accurately interpreted from fathometer data. The bottom 


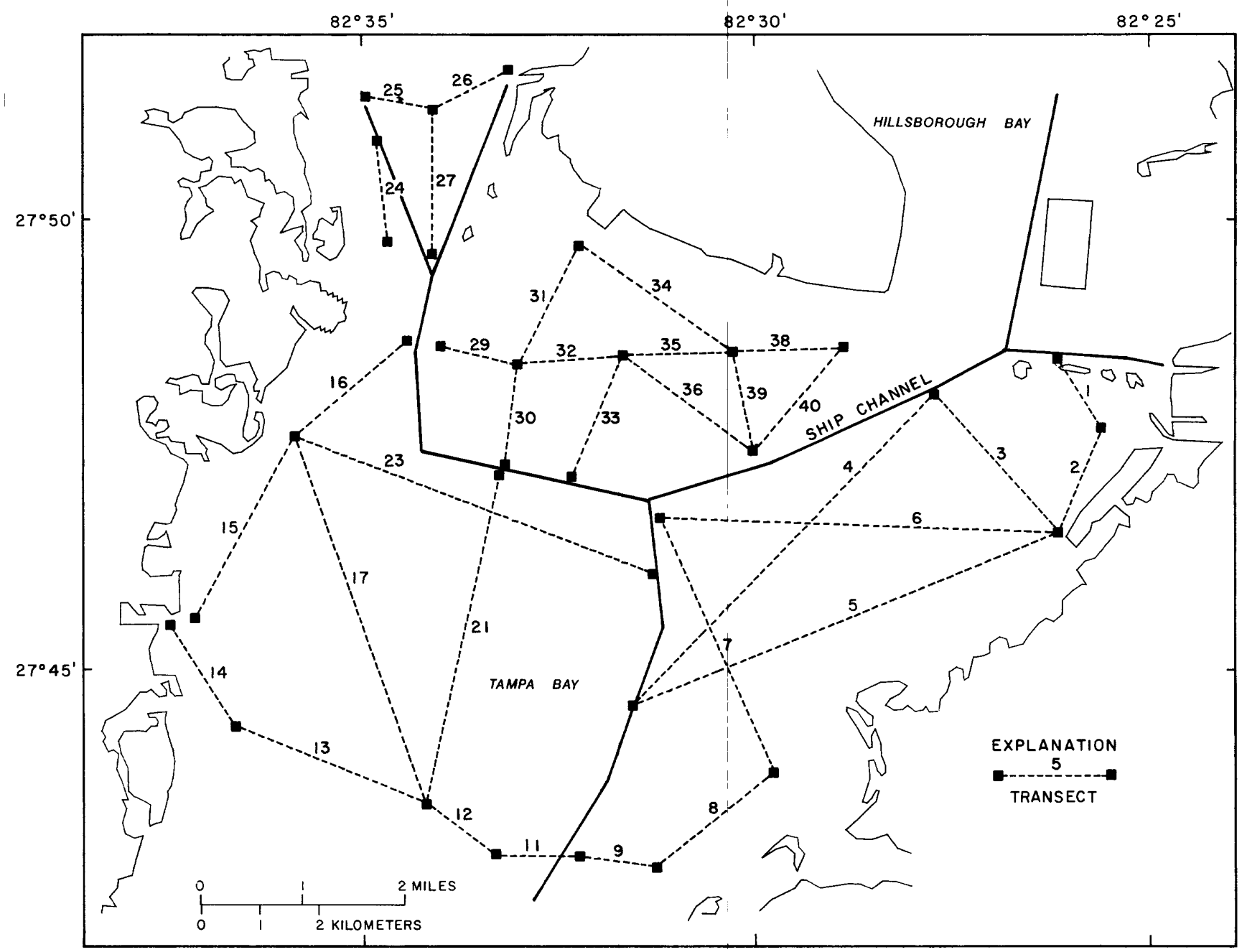

Figure 2. Fathometer transects in middle Tampa Bay. 


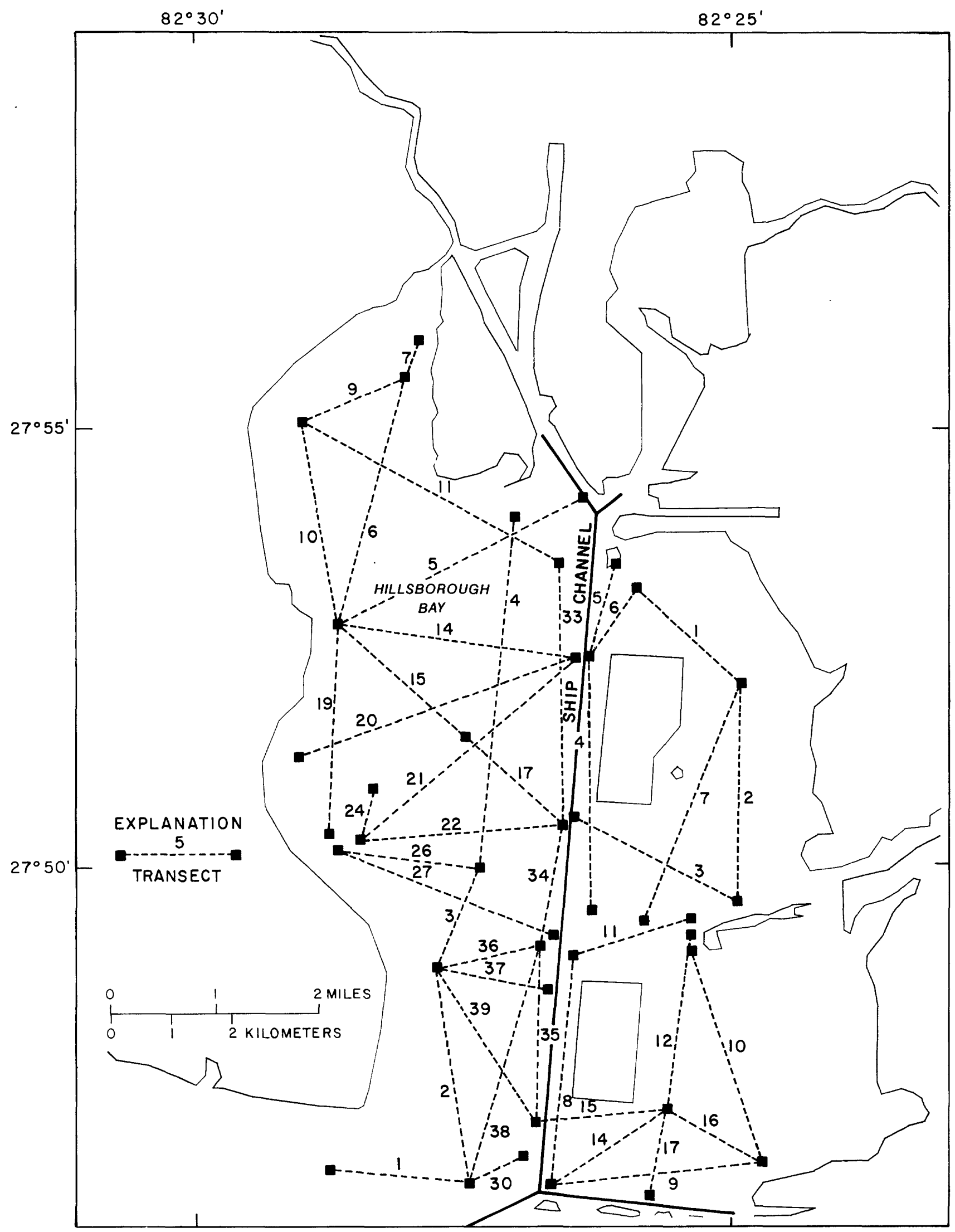

Figure 3. Fathometer transects in Hillsborough Bay. 


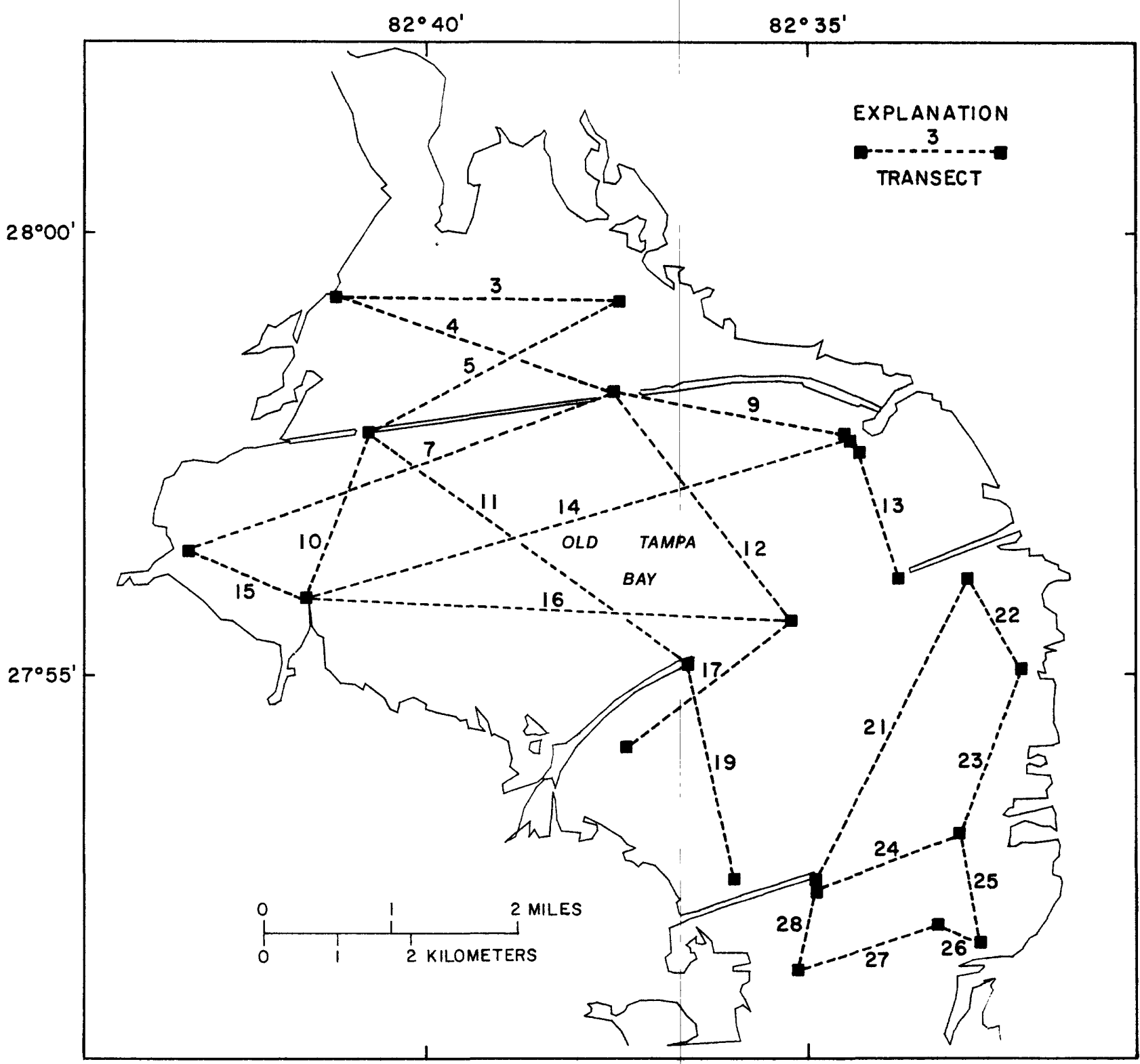

Figure 4. Fathometer transects in Old Tampa Bay.

Table 10. Grab sample and interpreted fathometer classifications

\begin{tabular}{cll}
\hline \multicolumn{1}{c}{$\begin{array}{c}\text { Classification } \\
\text { criteria }\end{array}$} & $\begin{array}{l}\text { Fathometer } \\
\text { classification }\end{array}$ & \multicolumn{1}{c}{$\begin{array}{c}\text { Grab sample } \\
\text { classification }\end{array}$} \\
\hline $0-12$ percent fines by weight & Coarse & Coarse \\
$12-50$ percent fines by weight & Coarse with fines & Coarse with fines \\
$50-80$ percent fines by weight & Fine & Fine with coarse material \\
$80-100$ percent fines by weight & Fine & Fine \\
$\begin{array}{l}\text { Very irregular bathymetry near } \\
\text { dredge fill sites }\end{array}$ & Spoil & \\
\hline
\end{tabular}


sediment in these areas generally contained a mixture of coarse and fine sediments, but was classified only as "spoil." A more detailed classification of sediments in the spoil areas was not deemed necessary for purposes of locating monitoring sites.

An example fathometer output for transect 30 in middle Tampa Bay is shown in figure 5. The water surface is indicated by the line at the top of the strip chart and the bottom is indicated by the darkest line 1 to $2 \mathrm{in}$. below. Each vertical division is equal to $1 \mathrm{ft}$ and the chart speed was set at $1 \mathrm{in} / \mathrm{min}$. The transect starts in about $5 \mathrm{ft}$ of water, which quickly increases to a depth of 10 to $12 \mathrm{ft}$ with sandy bottom sediments. One large and several small clusters of lines between the water surface and bottom are probably schools of fish. Just below the bottom signal is a second signal that is probably caused by harder material under the surficial sand. The lowest signal is the second reflectance off the bottom, which is initially strong in the shallow water, but becomes weak as the depth increases to 10 to $12 \mathrm{ft}$. Two minutes after the transect was started, the Loran-C position was $27^{\circ} 48^{\prime} 19^{\prime \prime} \mathrm{N}$ and $82^{\circ} 33^{\prime} 09^{\prime \prime} \mathrm{W}$. At about the 6-minute elapsed time mark, the depth increases to about $15 \mathrm{ft}$, and at about 7 minutes, the depth was about $20 \mathrm{ft}$. The second reflectance from this bottom slope is stronger, probably due either to coarser material on the slope or increased reflectance caused by the slope. Two spoil mounds are observed near the 8-minute mark. During the last 2 minutes of the transect, the bottom material consists of fine sediments. The edge of a shipping channel is apparent at the end of the transect at an elapsed time of 10 minutes 15 seconds. The initial bottom signal was the only signal recorded for the fine material. The thickness of the bottom signal increased in the area of finer material because the signal can penetrate deeper in fine sediments than in hard or sandy bottom materials.

Another example is part of transect 10 in west Hillsborough Bay shown in figure 6. The transect starts in about $8 \mathrm{ft}$ of water with a bottom material of coarse with fines (12- to 50-percent fines), as confirmed by a grab sample. The strength of the second reflected signal off the bed decreases with time as the fine sediments become more abundant. The high setting of the fathometer sensitivity caused noise that is present below the second reflected signal. A grab sample at the 5-minute mark contained fine with coarse material (greater than 50-percent fines). Slightly before the 3-minute mark, the second reflected signal fades, so this is assumed to be the division between predominantly coarse and predominantly fine material.

\section{Grab Samples}

Grab samples of bed material were collected to relate the fathometer data to sediment size classifications. Samples were collected at some starting and stopping points and from major bed features identified from the fathometer transects. Most of the samples were collected from the bed surface with an Ekman dredge, but samples in water deeper than 15 to $20 \mathrm{ft}$ were collected with a Ponar dredge. Both dredges usually closed well so that little, if any, fine material was lost. Standard field techniques were used to classify samples in which the amount of coarse particles is determined visually and by the grittiness of the sample (Foth, 1978). Samples that were difficult to classify in the field were saved and sieved in a laboratory. The sediments were classified as coarse, coarse with fines, fine with coarse material, or fine, as described in table 10. The fathometer data were not precise enough to differentiate between the grab sample classifications of fine with coarse material and fine, so the fine classification interpreted from the fathometer data includes the fine with coarse material and fine material as determined from the grab samples.

The first batch of samples that were collected and saved between October 1987 and February 1988 was sent to the U.S. Geological Survey sediment laboratory in Baton Rouge, La., for analysis and was accidentally dry sieved instead of wet sieved. During the drying process, fine sediments form aggregates that are difficult to break apart by mechanical sieving. The size distribution resulting from the dry sieving is thus coarser than the actual size distribution. The samples that had been dried were rewetted and wet sieved. At three test sites, the particle size distributions of the rewetted samples were nearly identical to particle size distributions of additional samples that were wet sieved. Thus, particle size classification of the rewetted samples were considered satisfactory for purposes of this study. All samples collected after February 1988 were wet sieved by the Geology Department at the University of South Florida.

Many of the sediment samples that were collected had a mean particle size near the $62.5-\mu \mathrm{m}$ division between the coarse and fine classifications and were poorly sorted (homogeneous particle size). Field identification of these borderline sediments was difficult, but improved with experience and with feedback from the laboratory analyses. Thirteen grab samples from the final collection trips in May 1989 that were the most difficult to classify in the field were saved and analyzed by the laboratory to confirm the field identification. All but one of the samples had been correctly classified in the field. Two previous sample batches sent to the laboratories for analysis indicated that the field classification often mistakenly identified very fine sand (between 62.5 and $125 \mu \mathrm{m}$ ) as fine material. Because of the initial problems of differentiating between very fine sand and fine material in the field, the grab samples considered in the analysis of the data were limited to: samples wet sieved by a laboratory (including rewetted samples); three Hillsborough Bay samples that were dry sieved, but contained substantial amounts of fine material so that reliable classification was still possible; samples classified as coarse material in field identifications; and all samples classified in the field during the final collection trips in May 1989. Thus, inclusion of potentially erroneous data in the analysis were minimized. 


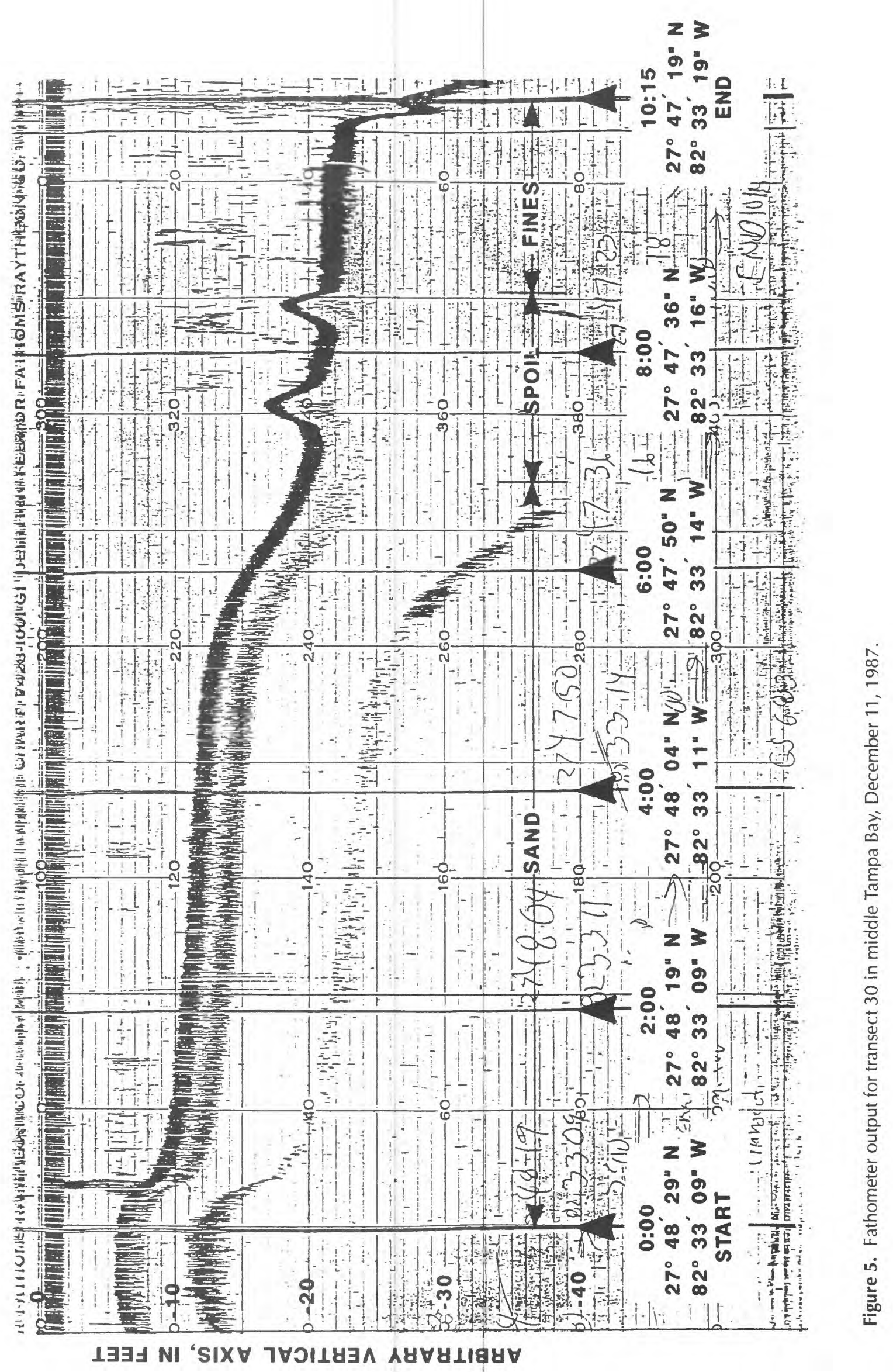




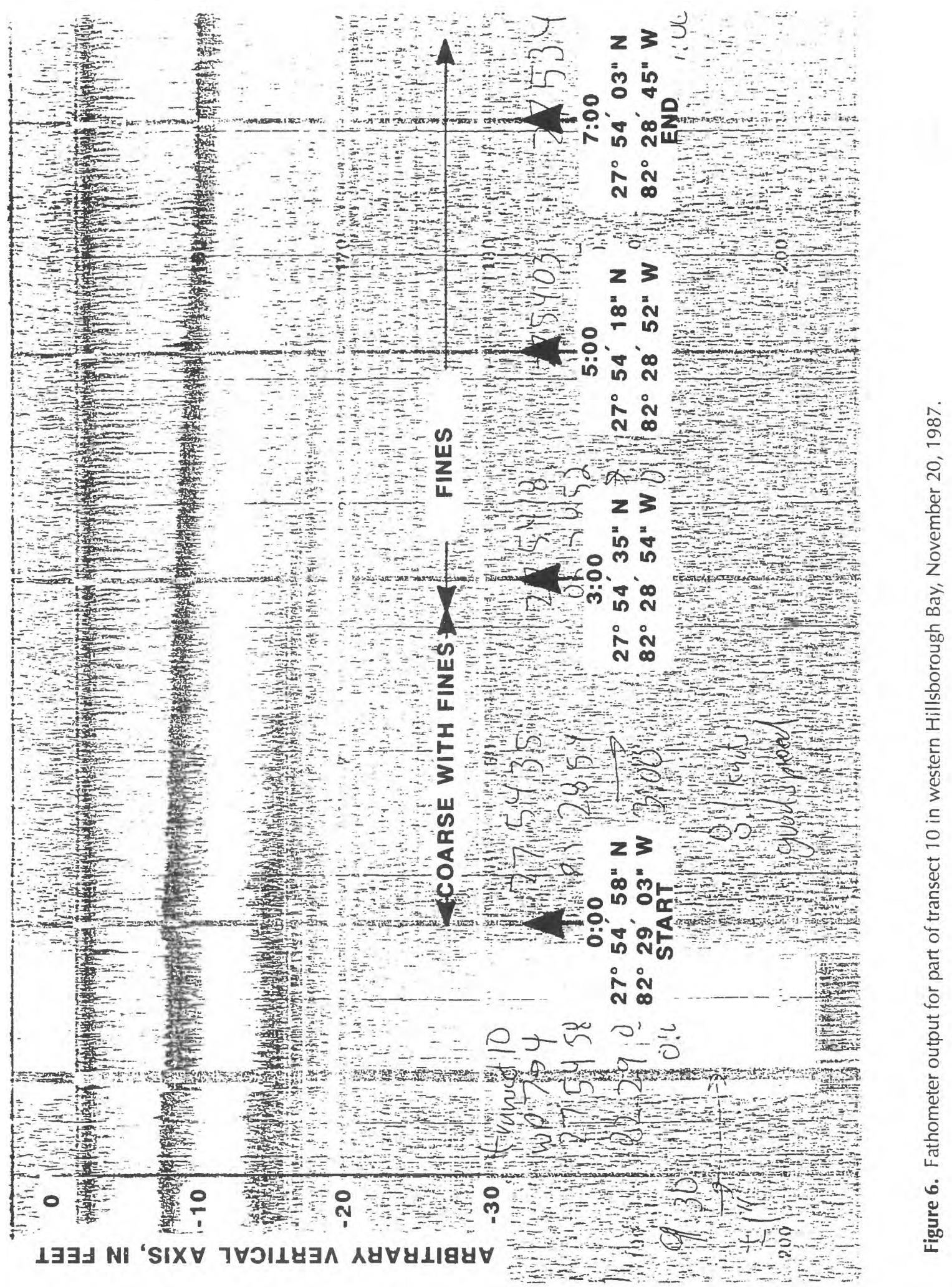




\section{SIZE CLASSIFICATION OF BED SEDIMENT}

The classification of bed sediments interpreted from fathometer and grab sample data are presented in figures 7 , 8 , and 9 for middle Tampa Bay, Hillsborough Bay, and Old Tampa Bay, respectively. The shaded areas in the figures represent areas of the bay bottom at which sediments with greater than 50-percent fine material either exist or are expected to exist based upon sediment classification data and bathymetric data. Shading is included to help visualize the probable extent of identified areas of fine sediment for the purpose of this study. Areas with greater than 50-percent fine material that were not identified by the garb sample or transect data, such as a small hole filled with fine sediment or a fine sediment bed in water less than $3 \mathrm{ft}$ deep, are not shaded in figures 7 through 9 .

The location of the interpreted fathometer classifications of the bottom sediment (coarse, coarse with fines, fine, and spoil) as a percentage of the distance from the starting nodes to the stopping nodes of the transects for middle Tampa Bay, Hillsborough Bay east of the ship channel, Hillsborough Bay west of the ship channel, and Old Tampa Bay are listed in tables $6,7,8$, and 9 , respectively. For example, table 6 indicates that the bed material at transect 1 in middle Tampa Bay is coarse material for the first 25 percent of the transect and coarse material with fines in the final 75 percent of the transect. Thus, as presented in

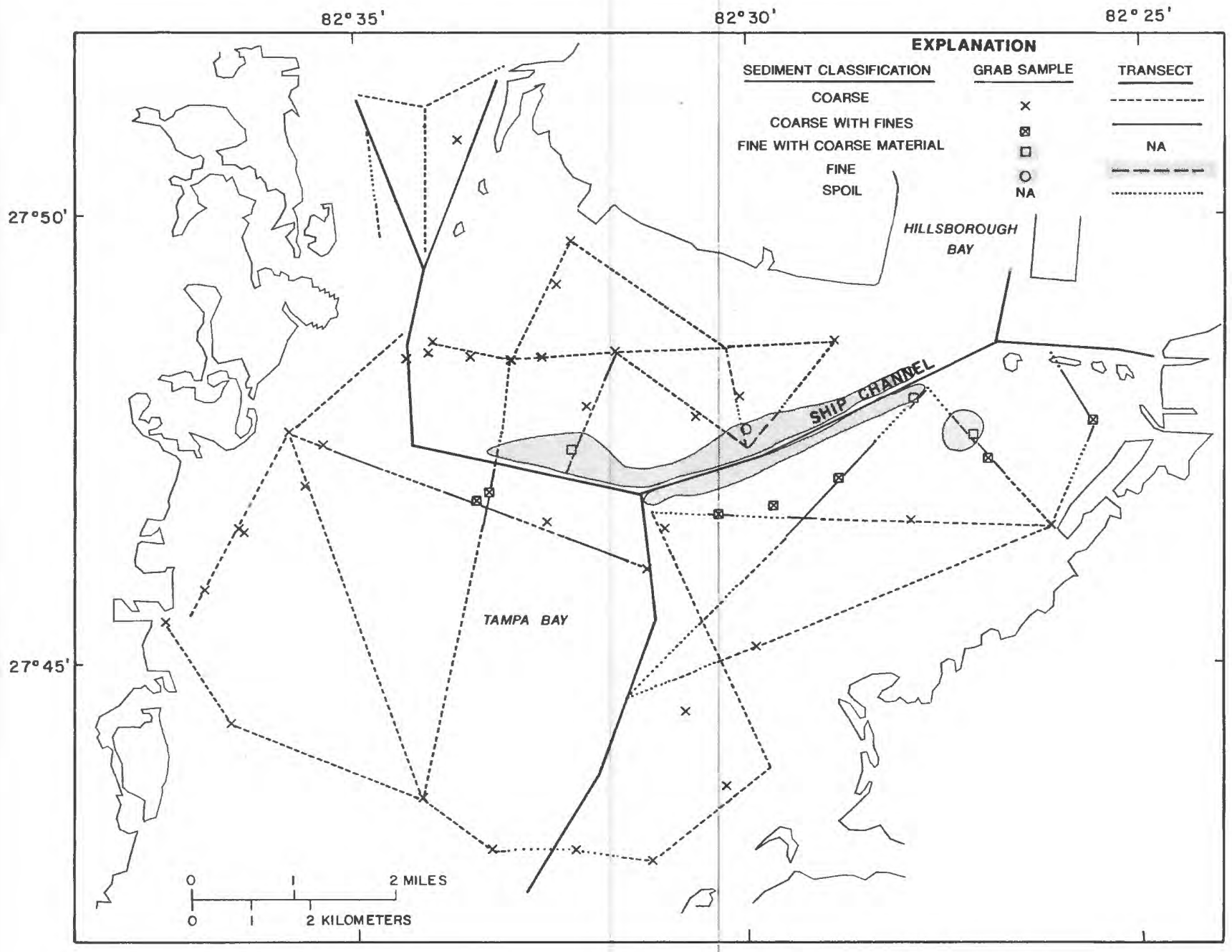

Figure 7. Sediment classification at transects in middle Tampa Bay, 1987-89. 


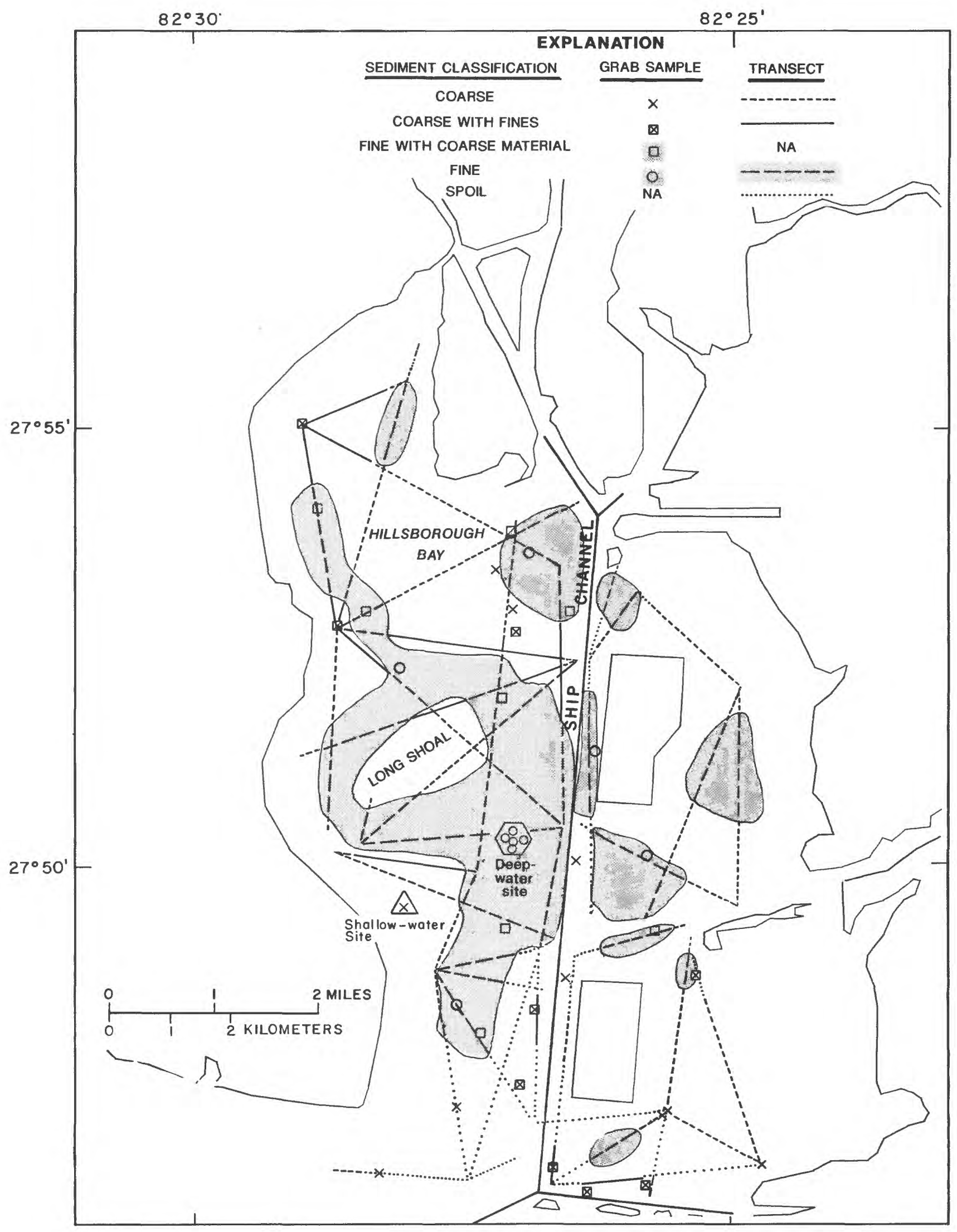

Figure 8. Sediment classification at transects in Hillsborough Bay, 1987-89. 


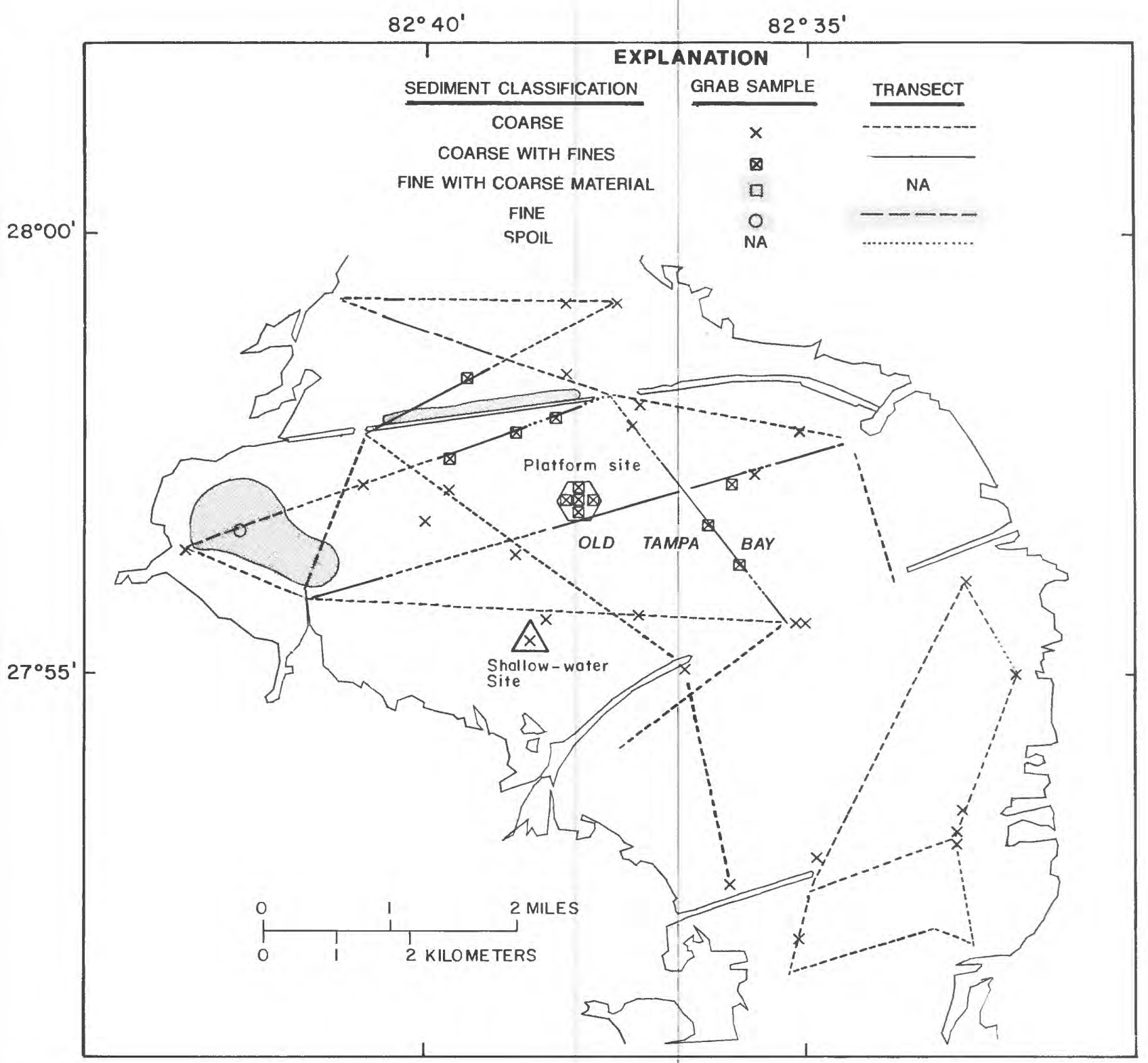

Figure 9. Sediment classification of transects in Old Tampa Bay, 1987-89.

table 2 , the spoil is near the starting node (node 2, $27^{\circ} 48^{\prime} 33^{\prime \prime} \mathrm{N}$ and $82^{\circ} 26^{\prime} 22^{\prime \prime} \mathrm{W}$ ), and the bottom sediment, near the stopping node (node $3,27^{\circ} 47^{\prime} 45^{\prime \prime} \mathrm{N}$ and $\left.82^{\circ} 25^{\prime} 49^{\prime \prime} \mathrm{W}\right)$, has a classification of coarse with fines.

The results of field laboratory analyses of grab samples are presented in tables 11, 12, and 13 for middle Tampa Bay, Hillsborough Bay, and Old Tampa Bay, respectively. For each sample, the tables list latitude; longitude; analysis method, either field, wet sieve, rewetted sieve, or dry sieve; and classification (as defined in table 10). For sieved samples, the mean particle diameter and percentage of fine material by weight are listed. Three dry-sieved samples from Hillsborough Bay are included in table 12 because a high percentage of fine material was found despite the probable underestimation of the fine material content by dry sieving. For some samples with more than 50-percent fine material, the particle size distribution of the fine fraction was not determined, so the mean particle diameter of the entire sample is unknown.

The sediment classifications are consistent with results of previous studies. Sediments are generally finer in Hillsborough Bay and in the deeper, more level parts of Tampa Bay, excluding ship channels. Sediments are coarser in shallower water and in middle Tampa Bay. In middle Tampa Bay, sandy bottom sediments are dominant, and the only sediments containing appreciable (greater than 12 percent by weight) fine material are adjacent to the ship channel at depths greater than $20 \mathrm{ft}$ and in some small 
Table 11. Results of analyses and classification of sediment grab samples collected from middle Tampa Bay, 1987-89

$[\mu \mathrm{m}$, microns; fines, particle diameter less than 62.5 microns; --, no data]

\begin{tabular}{|c|c|c|c|c|c|}
\hline \multicolumn{2}{|c|}{ Site location } & \multirow{2}{*}{$\begin{array}{c}\text { Analysis } \\
\text { method }\end{array}$} & \multirow{2}{*}{$\begin{array}{c}\text { Mean } \\
\text { diameter } \\
(\mu \mathrm{m})\end{array}$} & \multirow{2}{*}{$\begin{array}{l}\text { Per- } \\
\text { cent } \\
\text { fines }\end{array}$} & \multirow{2}{*}{$\begin{array}{c}\text { Sample } \\
\text { classification }\end{array}$} \\
\hline Latitude & Longitude & & & & \\
\hline $27^{\circ} 42^{\prime} 37^{\prime \prime} \mathrm{N}$ & $82^{\circ} 31^{\prime} 23^{\prime \prime} \mathrm{W}$ & Field & - & - & Coarse \\
\hline $27^{\circ} 42^{\prime} 44^{\prime \prime} \mathrm{N}$ & $82^{\circ} 32^{\prime} 21^{\prime \prime} \mathrm{W}$ & Field & -- & -- & Coarse \\
\hline $27^{\circ} 42^{\prime} 45^{\prime \prime} \mathrm{N}$ & $82^{\circ} 33^{\prime} 25^{\prime \prime} \mathrm{W}$ & Field & -- & -- & Coarse \\
\hline $27^{\circ} 43^{\prime} 22^{\prime \prime} \mathrm{N}$ & $82^{\circ} 34^{\prime} 18^{\prime \prime} \mathrm{W}$ & Field & -- & -- & Coarse \\
\hline $27^{\circ} 43^{\prime} 30^{\prime \prime} \mathrm{N}$ & $82^{\circ} 30^{\prime} 26^{\prime \prime} \mathrm{W}$ & Rewet & 144 & 8 & Coarse \\
\hline $27^{\circ} 44^{\prime} 16^{\prime \prime} \mathrm{N}$ & $82^{\circ} 36^{\prime} 44^{\prime \prime} \mathrm{W}$ & Field & -- & -- & Coarse \\
\hline $27^{\circ} 44^{\prime} 23^{\prime \prime} \mathrm{N}$ & $82^{\circ} 30^{\prime} 56^{\prime \prime} \mathrm{W}$ & Field & -- & -- & Coarse \\
\hline $27^{\circ} 45^{\prime} 10^{\prime \prime} \mathrm{N}$ & $82^{\circ} 30^{\prime} 02^{\prime \prime} \mathrm{W}$ & Field & -- & -- & Coarse \\
\hline $27^{\circ} 45^{\prime} 29^{\prime \prime} \mathrm{N}$ & $82^{\circ} 37^{\prime} 35^{\prime \prime} \mathrm{W}$ & Field & -- & -- & Coarse \\
\hline $27^{\circ} 45^{\prime} 49^{\prime \prime} \mathrm{N}$ & $82^{\circ} 37^{\prime} 05^{\prime \prime} \mathrm{W}$ & Field & -- & -- & Coarse \\
\hline $27^{\circ} 46^{\prime} 04^{\prime \prime} \mathrm{N}$ & $82^{\circ} 31^{\prime} 26^{\prime \prime} \mathrm{W}$ & Field & -- & -- & Coarse \\
\hline $27^{\circ} 46^{\prime} 30^{\prime \prime} \mathrm{N}$ & $82^{\circ} 36^{\prime} 34^{\prime \prime} \mathrm{W}$ & Wet & 126 & 5.6 & Coarse \\
\hline $27^{\circ} 46^{\prime} 32^{\prime \prime} \mathrm{N}$ & $82^{\circ} 31^{\prime} 13^{\prime \prime} \mathrm{W}$ & Field & -- & -- & Coarse \\
\hline $27^{\circ} 46^{\prime} 32^{\prime \prime} \mathrm{N}$ & $82^{\circ} 36^{\prime} 38^{\prime \prime} \mathrm{W}$ & Rewet & 144 & 7 & Coarse \\
\hline $27^{\circ} 46^{\prime} 33^{\prime \prime} \mathrm{N}$ & $82^{\circ} 26^{\prime} 21^{\prime \prime} \mathrm{W}$ & Field & -- & -- & Coarse \\
\hline $27^{\circ} 46^{\prime} 36^{\prime \prime} \mathrm{N}$ & $82^{\circ} 32^{\prime} 41^{\prime \prime} \mathrm{W}$ & Rewet & 164 & 8 & Coarse \\
\hline $27^{\circ} 46^{\prime} 37^{\prime \prime} \mathrm{N}$ & $82^{\circ} 28^{\prime} 05^{\prime \prime} \mathrm{W}$ & Field & -- & -- & Coarse \\
\hline $27^{\circ} 46^{\prime} 41^{\prime \prime} \mathrm{N}$ & $82^{\circ} 30^{\prime} 31^{\prime \prime} \mathrm{W}$ & Field & -- & -. & Coarse with fines \\
\hline $27^{\circ} 46^{\prime} 46^{\prime \prime} \mathrm{N}$ & $82^{\circ} 29^{\prime} 50^{\prime \prime} \mathrm{W}$ & Rewet & 151 & 15 & Coarse with fines \\
\hline $27^{\circ} 46^{\prime} 52^{\prime \prime} \mathrm{N}$ & $82^{\circ} 33^{\prime} 35^{\prime \prime} \mathrm{W}$ & Wet & 123 & 19.7 & Coarse with fines \\
\hline $27^{\circ} 46^{\prime} 57^{\prime \prime} \mathrm{N}$ & $82^{\circ} 33^{\prime} 26^{\prime \prime} \mathrm{W}$ & Field & -. & -- & Coarse with fines \\
\hline $27^{\circ} 47^{\prime} 03^{\prime \prime} \mathrm{N}$ & $82^{\circ} 35^{\prime} 46^{\prime \prime} \mathrm{W}$ & Wet & 110 & 11.1 & Coarse \\
\hline $27^{\circ} 47^{\prime} 06^{\prime \prime} \mathrm{N}$ & $82^{\circ} 29^{\prime} 00^{\prime \prime} \mathrm{W}$ & Wet & 149 & 20.1 & Coarse with fines \\
\hline $27^{\circ} 47^{\prime} 19^{\prime \prime} \mathrm{N}$ & $82^{\circ} 27^{\prime} 08^{\prime \prime} \mathrm{W}$ & Field & -- & -- & Coarse with fines \\
\hline $27^{\circ} 47^{\prime} 27^{\prime \prime} \mathrm{N}$ & $82^{\circ} 32^{\prime} 22^{\prime \prime} \mathrm{W}$ & Wet & 50 & 53.3 & Fine with coarse material \\
\hline $27^{\circ} 47^{\prime} 32^{\prime \prime} \mathrm{N}$ & $82^{\circ} 35^{\prime} 32^{\prime \prime} \mathrm{W}$ & Field & -- & - & Coarse \\
\hline $27^{\circ} 47^{\prime} 35^{\prime \prime} \mathrm{N}$ & $82^{\circ} 27^{\prime} 18^{\prime \prime} \mathrm{W}$ & Field & -- & -- & Fine with coarse material \\
\hline $27^{\circ} 47^{\prime} 40^{\prime \prime} \mathrm{N}$ & $82^{\circ} 30^{\prime} 10^{\prime \prime} \mathrm{W}$ & Field & -- & - & Fine \\
\hline $27^{\circ} 47^{\prime} 40^{\prime \prime} \mathrm{N}$ & $82^{\circ} 35^{\prime} 59^{\prime \prime} \mathrm{W}$ & Field & -- & -- & Coarse \\
\hline $27^{\circ} 47^{\prime} 45^{\prime \prime} \mathrm{N}$ & $82^{\circ} 25^{\prime} 49^{\prime \prime} \mathrm{W}$ & Rewet & 136 & 18 & Coarse with fines \\
\hline $27^{\circ} 47^{\prime} 49^{\prime \prime} \mathrm{N}$ & $82^{\circ} 30^{\prime} 48^{\prime \prime} \mathrm{W}$ & Field & -- & -- & Coarse \\
\hline $27^{\circ} 47^{\prime} 57^{\prime \prime} \mathrm{N}$ & $82^{\circ} 32^{\prime} 10^{\prime \prime} \mathrm{W}$ & Rewet & 236 & 8 & Coarse \\
\hline $27^{\circ} 48^{\prime} 01^{\prime \prime} \mathrm{N}$ & $82^{\circ} 28^{\prime} 03^{\prime \prime} \mathrm{W}$ & Wet & 56 & 51.5 & Fine with coarse material \\
\hline $27^{\circ} 48^{\prime} 03^{\prime \prime} \mathrm{N}$ & $82^{\circ} 30^{\prime} 15^{\prime \prime} \mathrm{W}$ & Field & -- & -- & Coarse \\
\hline $27^{\circ} 48^{\prime} 29^{\prime \prime} \mathrm{N}$ & $82^{\circ} 33^{\prime} 09^{\prime \prime} \mathrm{W}$ & Field & -- & -- & Coarse \\
\hline $27^{\circ} 48^{\prime} 30^{\prime \prime} \mathrm{N}$ & $82^{\circ} 32^{\prime} 46^{\prime \prime} \mathrm{W}$ & Field & -- & -- & Coarse \\
\hline $27^{\circ} 48^{\prime} 32^{\prime \prime} \mathrm{N}$ & $82^{\circ} 33^{\prime} 38^{\prime \prime} \mathrm{W}$ & Field & -- & $\ldots$ & Coarse \\
\hline $27^{\circ} 48^{\prime} 32^{\prime \prime} \mathrm{N}$ & $82^{\circ} 34^{\prime} 28^{\prime \prime} \mathrm{W}$ & Field & -- & -. & Coarse \\
\hline $27^{\circ} 48^{\prime} 35^{\prime \prime} \mathrm{N}$ & $82^{\circ} 31^{\prime} 49^{\prime \prime} \mathrm{W}$ & Field & -- & -- & Coarse \\
\hline $27^{\circ} 48^{\prime} 35^{\prime \prime} \mathrm{N}$ & $82^{\circ} 34^{\prime} 11^{\prime \prime} \mathrm{W}$ & Field & -- & -- & Coarse \\
\hline $27^{\circ} 48^{\prime} 42^{\prime \prime} \mathrm{N}$ & $82^{\circ} 29^{\prime} 02^{\prime \prime} \mathrm{W}$ & Rewet & 198 & 5 & Coarse \\
\hline $27^{\circ} 48^{\prime} 42^{\prime \prime} \mathrm{N}$ & $82^{\circ} 34^{\prime} 08^{\prime \prime} \mathrm{W}$ & Field & -- & -- & Coarse \\
\hline $27^{\circ} 49^{\prime} 21^{\prime \prime} \mathrm{N}$ & $82^{\circ} 32^{\prime} 33^{\prime \prime} \mathrm{W}$ & Field & -- & -- & Coarse \\
\hline $27^{\circ} 49^{\prime} 51^{\prime \prime} \mathrm{N}$ & $82^{\circ} 32^{\prime} 22^{\prime \prime} \mathrm{W}$ & Field & -- & -- & Coarse \\
\hline $27^{\circ} 51^{\prime} 02^{\prime \prime} \mathrm{N}$ & $82^{\circ} 33^{\prime} 47^{\prime \prime} \mathrm{W}$ & Field & -- & -- & Coarse \\
\hline
\end{tabular}


Table 12. Results of analyses and classification of sediment grab samples collected from Hillsborough Bay, 1987-89

[ $\mu \mathrm{m}$, microns; fines, particle diameter less than 62.5 microns; --, no data]

\begin{tabular}{|c|c|c|c|c|c|}
\hline \multicolumn{2}{|c|}{ Site location } & \multirow{2}{*}{$\begin{array}{l}\text { Analysis } \\
\text { method }\end{array}$} & \multirow{2}{*}{$\begin{array}{c}\text { Mean } \\
\text { diameter } \\
(\mu \mathrm{m})\end{array}$} & \multirow{2}{*}{$\begin{array}{l}\text { Per- } \\
\text { cent } \\
\text { fines }\end{array}$} & \multirow{2}{*}{$\begin{array}{c}\text { Sample } \\
\text { classification }\end{array}$} \\
\hline Latitude & Longitude & & & & \\
\hline $27^{\circ} 48^{\prime} 36^{\prime \prime} \mathrm{N}$ & $82^{\circ} 26^{\prime} 23^{\prime \prime} \mathrm{W}$ & Wet & 133 & 19.7 & Coarse with fines \\
\hline $27^{\circ} 48^{\prime} 40^{\prime \prime} \mathrm{N}$ & $82^{\circ} 25^{\prime} 52^{\prime \prime} \mathrm{W}$ & Field & -- & -- & Coarse with fines \\
\hline $27^{\circ} 48^{\prime} 47^{\prime \prime N}$ & $82^{\circ} 27^{\prime} 79^{\prime \prime} \mathrm{W}$ & Field & -- & -- & Coarse \\
\hline $27^{\circ} 48^{\prime} 49^{\prime \prime} \mathrm{N}$ & $82^{\circ} 26^{\prime} 43^{\prime \prime} \mathrm{W}$ & Field & - & -- & Coarse with fines \\
\hline $27^{\circ} 48^{\prime} 50^{\prime \prime} \mathrm{N}$ & $82^{\circ} 24^{\prime} 50^{\prime \prime} \mathrm{W}$ & Field & -- & -- & Coarse \\
\hline $27^{\circ} 49^{\prime} 14^{\prime \prime} \mathrm{N}$ & $82^{\circ} 25^{\prime} 44^{\prime \prime} \mathrm{W}$ & Field & -- & -- & Coarse \\
\hline $27^{\circ} 49^{\prime} 17^{\prime \prime N}$ & $82^{\circ} 25^{\prime} 42^{\prime \prime} \mathrm{W}$ & Field & -- & -- & Coarse \\
\hline $27^{\circ} 49^{\prime} 20^{\prime \prime} \mathrm{N}$ & $82^{\circ} 27^{\prime} 36^{\prime \prime W}$ & Field & -- & -- & Coarse \\
\hline $27^{\circ} 49^{\prime} 31^{\prime \prime} \mathrm{N}$ & $82^{\circ} 27^{\prime} 02^{\prime \prime W}$ & Rewet & 275 & 16 & Coarse with fines \\
\hline $27^{\circ} 49^{\prime} 57^{\prime \prime} \mathrm{N}$ & $82^{\circ} 27^{\prime} 23^{\prime \prime} \mathrm{W}$ & Field & -- & -- & Fine with coarse material \\
\hline $27^{\circ} 50^{\prime} 09^{\prime \prime} \mathrm{N}$ & $82^{\circ} 26^{\prime} 53^{\prime \prime} \mathrm{W}$ & Wet & 103 & 26.6 & Coarse with fines \\
\hline $27^{\circ} 50^{\prime} 10^{\prime \prime} \mathrm{N}$ & $82^{\circ} 27^{\prime} 34^{\prime \prime} \mathrm{W}$ & Field & -- & -- & Fine \\
\hline $27^{\circ} 50^{\prime} 24^{\prime \prime N}$ & $82^{\circ} 26^{\prime} 36^{\prime \prime} \mathrm{W}$ & Field & -- & -- & Coarse \\
\hline $27^{\circ} 50^{\prime} 26^{\prime \prime} \mathrm{N}$ & $82^{\circ} 25^{\prime} 25^{\prime \prime} \mathrm{W}$ & Field & - & -- & Coarse with fines \\
\hline $27^{\circ} 50^{\prime} 48^{\prime \prime} \mathrm{N}$ & $82^{\circ} 25^{\prime} 47^{\prime \prime} \mathrm{W}$ & Field & -- & -- & Fine with coarse material \\
\hline $27^{\circ} 50^{\prime} 50^{\prime \prime} \mathrm{N}$ & $82^{\circ} 27^{\prime} 09^{\prime \prime} \mathrm{W}$ & Wet & 20 & 73.0 & Fine with coarse material \\
\hline $27^{\circ} 51^{\prime} 00^{\prime \prime N}$ & $82^{\circ} 28^{\prime} 05^{\prime \prime} \mathrm{W}$ & Rewet & 166 & 2 & Coarse \\
\hline $27^{\circ} 51^{\prime} 23 " \mathrm{~N}$ & $82^{\circ} 26^{\prime} 29^{\prime \prime} \mathrm{W}$ & Rewet & 145 & 9 & Coarse \\
\hline $27^{\circ} 51^{\prime} 25^{\prime \prime} \mathrm{N}$ & $82^{\circ} 25^{\prime} 52^{\prime \prime} \mathrm{W}$ & Field & -- &.- & Fine \\
\hline $27^{\circ} 51^{\prime} 30^{\prime \prime} \mathrm{N}$ & $82^{\circ} 27^{\prime} 05^{\prime \prime} \mathrm{W}$ & Rewet & -- & 89 & Fine \\
\hline $27^{\circ} 51^{\prime} 34^{\prime \prime} \mathrm{N}$ & $82^{\circ} 27^{\prime} 00 " \mathrm{~W}$ & Rewet & -- & 90 & Fine \\
\hline $27^{\circ} 51^{\prime} 34^{\prime \prime N}$ & $82^{\circ} 27^{\prime} 05^{\prime \prime} \mathrm{W}$ & Wet & -- & 88.4 & Fine \\
\hline $27^{\circ} 51^{\prime} 36^{\prime \prime} \mathrm{N}$ & $82^{\circ} 27^{\prime} 09^{\prime \prime} \mathrm{W}$ & Rewet & -- & 83 & Fine \\
\hline $27^{\circ} 51^{\prime} 39^{\prime \prime} \mathrm{N}$ & $82^{\circ} 27^{\prime} 05^{\prime \prime} \mathrm{W}$ & Rewet & -. & 84 & Fine \\
\hline $27^{\circ} 52^{\prime} 17^{\prime \prime} \mathrm{N}$ & $82^{\circ} 26^{\prime} 21^{\prime \prime W}$ & Wet & 10 & 90.7 & Fine \\
\hline $27^{\circ} 52^{\prime} 45^{\prime \prime} \mathrm{N}$ & $82^{\circ} 27^{\prime} 11 " \mathrm{~W}$ & Dry & -- & 55 & Fine with coarse material \\
\hline $27^{\circ} 53^{\prime} 00^{\prime \prime} \mathrm{N}$ & $82^{\circ} 28^{\prime} 04^{\prime \prime} \mathrm{W}$ & Rewet & -- & 82 & Fine \\
\hline $27^{\circ} 53^{\prime} 18^{\prime \prime} \mathrm{N}$ & $82^{\circ} 27^{\prime} 03^{\prime \prime} \mathrm{W}$ & Rewet & 177 & 15 & Coarse with fines \\
\hline $27^{\circ} 53^{\prime} 20^{\prime \prime} \mathrm{N}$ & $82^{\circ} 28^{\prime} 41^{\prime \prime} \mathrm{W}$ & Rewet & 151 & 22 & Coarse with fines \\
\hline $27^{\circ} 53^{\prime} 27^{\prime \prime} \mathrm{N}$ & $82^{\circ} 26^{\prime} 33^{\prime \prime} \mathrm{W}$ & Field & -- & -- & Fine with coarse material \\
\hline $27^{\circ} 53^{\prime} 27^{\prime \prime N}$ & $82^{\circ} 28^{\prime} 25^{\prime \prime} \mathrm{W}$ & Wet & 35 & 53.6 & Fine with coarse material \\
\hline $27^{\circ} 53^{\prime} 29^{\prime \prime} \mathrm{N}$ & $82^{\circ} 27^{\prime} 05^{\prime \prime W}$ & Field & -- & -- & Coarse \\
\hline $27^{\circ} 53^{\prime} 49^{\prime \prime} \mathrm{N}$ & $82^{\circ} 27^{\prime} 14^{\prime \prime} \mathrm{W}$ & Field & -- & -- & Coarse \\
\hline $27^{\circ} 53^{\prime} 57^{\prime \prime} \mathrm{N}$ & $82^{\circ} 26^{\prime} 56^{\prime \prime W}$ & Dry & -- & 84 & Fine \\
\hline $27^{\circ} 54^{\prime} 05^{\prime \prime} \mathrm{N}$ & $82^{\circ} 27^{\prime} 05^{\prime \prime} \mathrm{W}$ & Dry & -- & 66 & Fine with coarse material \\
\hline $27^{\circ} 54^{\prime} 18^{\prime \prime N}$ & $82^{\circ} 28^{\prime} 52^{\prime \prime} \mathrm{W}$ & Field & -- & -- & Fine with coarse material \\
\hline $27^{\circ} 55^{\prime} 00^{\prime \prime} \mathrm{N}$ & $82^{\circ} 29^{\prime} 00^{\prime \prime} \mathrm{W}$ & Rewet & 94 & 34 & Coarse with fines \\
\hline
\end{tabular}


Table 13. Results of analyses and classification of sediment grab samples collected from Old Tampa Bay, 1987-89

[ $\mu \mathrm{m}$, microns; fines, particle diameter less than 62.5 microns; --, no data]

\begin{tabular}{|c|c|c|c|c|c|}
\hline \multicolumn{2}{|c|}{ Site location } & \multirow{2}{*}{$\begin{array}{c}\text { Analysis } \\
\text { method }\end{array}$} & \multirow{2}{*}{$\begin{array}{c}\text { Mean } \\
\text { diameter } \\
(\mu \mathrm{m})\end{array}$} & \multirow{2}{*}{$\begin{array}{l}\text { Per- } \\
\text { cent } \\
\text { fines }\end{array}$} & \multirow{2}{*}{$\begin{array}{c}\text { Sample } \\
\text { classification }\end{array}$} \\
\hline Latitude & Longitude & & & & \\
\hline $27^{\circ} 52^{\prime} 07^{\prime \prime N}$ & $82^{\circ} 35^{\prime} 05^{\prime \prime} \mathrm{W}$ & Field & -- & -- & Coarse \\
\hline $27^{\circ} 52^{\prime} 45^{\prime \prime} \mathrm{N}$ & $82^{\circ} 35^{\prime} 59^{\prime \prime} \mathrm{W}$ & Field & -- & -- & Coarse \\
\hline $27^{\circ} 53^{\prime} 02^{\prime \prime} \mathrm{N}$ & $82^{\circ} 34^{\prime} 52^{\prime \prime} \mathrm{W}$ & Field & -- & -- & Coarse \\
\hline $27^{\circ} 53^{\prime} 14^{\prime \prime} \mathrm{N}$ & $82^{\circ} 33^{\prime} 05^{\prime \prime W}$ & Field & -- & -- & Coarse \\
\hline $27^{\circ} 53^{\prime} 18^{\prime \prime} \mathrm{N}$ & $82^{\circ} 33^{\prime} 06^{\prime \prime} \mathrm{W}$ & Field & -- & -- & Coarse \\
\hline $27^{\circ} 53^{\prime} 34^{\prime \prime N}$ & $82^{\circ} 33^{\prime} 01 " \mathrm{~W}$ & Field & -- & -- & Coarse \\
\hline $27^{\circ} 55^{\prime} 05^{\prime \prime} \mathrm{N}$ & $82^{\circ} 32^{\prime} 19^{\prime \prime} \mathrm{W}$ & Field & -- & -- & Coarse \\
\hline $27^{\circ} 55^{\prime} 11^{\prime \prime} \mathrm{N}$ & $82^{\circ} 36^{\prime} 34^{\prime \prime} \mathrm{W}$ & Field & -- & -- & Coarse \\
\hline $27^{\circ} 55^{\prime} 30^{\prime \prime} \mathrm{N}$ & $82^{\circ} 38^{\prime} 33^{\prime \prime} \mathrm{W}$ & Wet & 152 & 1.5 & Coarse \\
\hline $27^{\circ} 55^{\prime} 39^{\prime \prime} \mathrm{N}$ & $82^{\circ} 35^{\prime} 08^{\prime \prime} \mathrm{W}$ & Field & -- & -- & Coarse \\
\hline $27^{\circ} 55^{\prime} 39^{\prime \prime} \mathrm{N}$ & $82^{\circ} 35^{\prime} 14^{\prime \prime} \mathrm{W}$ & Field & -- & -- & Coarse \\
\hline $27^{\circ} 55^{\prime} 44^{\prime \prime} \mathrm{N}$ & $82^{\circ} 38^{\prime} 20^{\prime \prime} \mathrm{W}$ & Rewet & 154 & 4 & Coarse \\
\hline $27^{\circ} 55^{\prime} 46^{\prime \prime} \mathrm{N}$ & $82^{\circ} 37^{\prime} 09^{\prime \prime W}$ & Rewet & 143 & 11 & Coarse \\
\hline $27^{\circ} 56^{\prime} 08^{\prime \prime N}$ & $82^{\circ} 32^{\prime} 57^{\prime \prime} \mathrm{W}$ & Field & -- & -- & Coarse \\
\hline $27^{\circ} 56^{\prime} 19^{\prime \prime} \mathrm{N}$ & $82^{\circ} 35^{\prime} 51^{\prime \prime} \mathrm{W}$ & Rewet & 145 & 12.2 & Coarse with fines \\
\hline $27^{\circ} 56^{\prime} 28^{\prime \prime N} \mathrm{~N}$ & $82^{\circ} 38^{\prime} 43^{\prime \prime} \mathrm{W}$ & Field & -- & -- & Coarse \\
\hline $27^{\circ} 56^{\prime} 30^{\prime \prime} \mathrm{N}$ & $82^{\circ} 43^{\prime} 02^{\prime \prime} \mathrm{W}$ & Field & -- & -- & Coarse \\
\hline $27^{\circ} 56^{\prime} 41 " \mathrm{~N}$ & $82^{\circ} 42^{\prime} 19^{\prime \prime} \mathrm{W}$ & Field & -- & -- & Fine \\
\hline $27^{\circ} 56^{\prime} 46^{\prime \prime} \mathrm{N}$ & $82^{\circ} 36^{\prime} 13^{\prime \prime} \mathrm{W}$ & Wet & 115 & 14.3 & Coarse with fines \\
\hline $27^{\circ} 56^{\prime} 49^{\prime \prime} \mathrm{N}$ & $82^{\circ} 39^{\prime} 54^{\prime \prime} \mathrm{W}$ & Rewet & 139 & 11 & Coarse \\
\hline $27^{\circ} 56^{\prime} 59^{\prime \prime} \mathrm{N}$ & $82^{\circ} 37^{\prime} 56^{\prime \prime W}$ & Rewet & 152 & 13 & Coarse with fines \\
\hline $27^{\circ} 57^{\prime} 01 " \mathrm{~N}$ & $82^{\circ} 37^{\prime} 52^{\prime \prime W}$ & Rewet & 137 & 17 & Coarse with fines \\
\hline $27^{\circ} 57^{\prime} 01^{\prime \prime N}$ & $82^{\circ} 37^{\prime} 55^{\prime \prime} \mathrm{W}$ & Wet & 127 & 16.3 & Coarse with fines \\
\hline $27^{\circ} 57^{\prime} 01^{\prime \prime N}$ & $82^{\circ} 37^{\prime} 59^{\prime \prime} \mathrm{W}$ & Rewet & 149 & 19 & Coarse with fines \\
\hline $27^{\circ} 57^{\prime} 03^{\prime \prime} \mathrm{N}$ & $82^{\circ} 37^{\prime} 54^{\prime \prime W}$ & Rewet & 149 & 14 & Coarse with fines \\
\hline $27^{\circ} 57^{\prime} 10^{\prime \prime} \mathrm{N}$ & $82^{\circ} 39^{\prime} 37^{\prime \prime} \mathrm{W}$ & Rewet & 144 & 10 & Coarse \\
\hline $27^{\circ} 57^{\prime} 12^{\prime \prime} \mathrm{N}$ & $82^{\circ} 40^{\prime} 43^{\prime \prime} \mathrm{W}$ & Rewet & 139 & 11 & Coarse \\
\hline $27^{\circ} 57^{\prime} 13^{\prime \prime N} \mathrm{~N}$ & $82^{\circ} 35^{\prime} 56^{\prime \prime W}$ & Field & -- & - & Coarse with fines \\
\hline $27^{\circ} 57^{\prime} 19^{\prime \prime} \mathrm{N}$ & $82^{\circ} 35^{\prime} 39^{\prime \prime} \mathrm{W}$ & Field & -- & -- & Coarse \\
\hline $27^{\circ} 57^{\prime} 31^{\prime \prime} \mathrm{N}$ & $82^{\circ} 39^{\prime} 35^{\prime \prime} \mathrm{W}$ & Wet & 131 & 20.5 & Coarse with fines \\
\hline $27^{\circ} 57^{\prime} 47^{\prime \prime N}$ & $82^{\circ} 35^{\prime} 05^{\prime \prime} \mathrm{W}$ & Rewet & 124 & 9 & Coarse \\
\hline $27^{\circ} 57^{\prime} 47^{\prime \prime} \mathrm{N}$ & $82^{\circ} 38^{\prime} 43^{\prime \prime} \mathrm{W}$ & Wet & 103 & 22.8 & Coarse with fines \\
\hline $27^{\circ} 57^{\prime} 52^{\prime \prime N}$ & $82^{\circ} 37^{\prime} 14^{\prime \prime W}$ & Field & -- & -- & Coarse \\
\hline $27^{\circ} 57^{\prime} 57^{\prime \prime N} \mathrm{~N}$ & $82^{\circ} 38^{\prime} 13^{\prime \prime} \mathrm{W}$ & Field & -- & -- & Coarse with fines \\
\hline $27^{\circ} 58^{\prime} 04^{\prime \prime} \mathrm{N}$ & $82^{\circ} 37^{\prime} 08^{\prime \prime W}$ & Field & -- & -- & Coarse \\
\hline $27^{\circ} 58^{\prime} 23^{\prime \prime N}$ & $82^{\circ} 39^{\prime} 20^{\prime \prime} \mathrm{W}$ & Wet & 110 & 16.1 & Coarse with fines \\
\hline $27^{\circ} 58^{\prime} 25^{\prime \prime} \mathrm{N}$ & $82^{\circ} 38^{\prime} 05^{\prime \prime} \mathrm{W}$ & Field & -- & -- & Coarse \\
\hline $27^{\circ} 59^{\prime} 11 " \mathrm{~N}$ & $82^{\circ} 38^{\prime} 05^{\prime \prime} \mathrm{W}$ & Field & -- & -- & Coarse \\
\hline $27^{\circ} 59^{\prime} 12^{\prime \prime} \mathrm{N}$ & $82^{\circ} 37^{\prime} 25^{\prime \prime W}$ & Field & -- & -- & Coarse \\
\hline
\end{tabular}


depressions at depths greater than $15 \mathrm{ft}$. Most of Hillsborough Bay has appreciable fine material, and the abundance of fine material generally increases with water depth. Two isolated pockets of fine sediment were identified in Old Tampa Bay east of Clearwater and north of the Courtney Campbell Causeway, and a large part of the area between the Courtney Campbell Causeway and the Howard Frankland Bridge greater than $12 \mathrm{ft}$ deep has appreciable fine material.

\section{SELECTION OF RESUSPENSION MONITORING SITES}

Sediment resuspension can not be simultaneously monitored throughout upper Tampa Bay, so representative sites for resuspension monitoring were selected. The most important selection criterion used was that a potential site be at the center of a large area of homogeneous sediment. Selection of a site in the center of a large homogeneous area of bed sediments ensures that the site is representative of a large fraction of the bay bottom and minimizes advective effects such as finer sediments nearby being resuspended, being carried to the site by the current, and being mistaken for resuspension of sediment actually present at the monitoring site. Finer sediments generally are present in the deeper parts of the bay and are lighter and, therefore, more easily suspended. Coarser sediments closer to shore in shallower water may be subjected to more wave activity, however, and also are likely to be resuspended. Thus, selection of a deeper site with finer sediments and a shallow site with coarser sediments in middle Tampa Bay, Old Tampa Bay, and Hillsborough Bay was an objective of the site selection process. Other criteria included nearly uniform residual currents over the homogeneous sediment bed (Goodwin, 1987), location far from ship channels for safety, and a secluded location to reduce vandalism.

Two monitoring sites were selected in areas of fine and coarse (sandy) bed sediments in Old Tampa Bay. The largest area of bottom sediments with appreciable fine material in Old Tampa Bay is between the Courtney Campbell Causeway and the Howard Frankland Bridge. This area is approximately $3 \mathrm{mi}$ long and as much as $1 \mathrm{mi}$ wide. A proposed resuspension monitoring site was located approximately at the center of this area, at latitude $27^{\circ} 57^{\prime} 01^{\prime \prime}$ $\mathrm{N}$ and longitude $82^{\circ} 37^{\prime} 55^{\prime \prime} \mathrm{W}$ (from Loran-C) (fig. 9). The water depth at this site (platform site) is about $12 \mathrm{ft}$. Grab samples were collected at the site and within about $100 \mathrm{yd}$ of the site to verify that the sediments were homogeneous in the vicinity of the site. The bottom material is approximately 16 percent fines and 2.73 percent organic carbon. An instrument platform was constructed at the site in June 1988 (Schoellhamer, 1990). A shallow-water site with a sandy bed and an average depth of approximately $4 \mathrm{ft}$ was located in Old Tampa Bay at latitude $27^{\circ} 55^{\prime} 30^{\prime \prime} \mathrm{N}$ and longitude $82^{\circ} 38^{\prime} 33^{\prime \prime}$ W (from Loran-C, see fig. 9). This site will be used for taking comparative water-quality measurements and for occasional deployment of a portable resuspension monitoring instrument package during the resuspension study.

Two sediment resuspension monitoring sites also were selected in Hillsborough Bay. The bottom sediments in the deeper parts of Hillsborough Bay are almost exclusively fine material, but these areas are separated by shoals, ship channels, and spoil piles. The largest and most representative area of fine bottom material in Hillsborough Bay is west of the ship channel and south of Long Shoal (fig. 8). A resuspension monitoring site was located at the approximate center of this area. Additional sampling in the vicinity confirmed that the bottom material is homogeneous and is approximately 88 percent fine material. This site is at latitude $27^{\circ} 51^{\prime} 34^{\prime \prime} \mathrm{N}$ and longitude $82^{\circ} 27^{\prime} 05^{\prime \prime} \mathrm{W}$ (from Loran- $\mathrm{C}$ ), as shown in figure 8 . A shallow-water site for comparative water-quality measurements and occasional resuspension monitoring with a portable instrument package was located in an area of Hillsborough Bay with sandy bottom material where the average depth was about $3 \mathrm{ft}$. The shallow-water site is located at latitude $27^{\circ} 51^{\prime} 00^{\prime \prime} \mathrm{N}$ and longitude $82^{\circ} 28^{\prime} 05^{\prime \prime} \mathrm{W}$ (from Loran-C), as shown in figure 8 .

In middle Tampa Bay, the largest area of bottom sediments with appreciable fine material was south of the ship channel that enters Hillsborough Bay (fig. 7). This area is approximately $0.5 \mathrm{mi}$ wide and is bounded to the north by the ship channel and spoil areas. The bottom material in this area is primarily coarse with fines. Because the selected area of coarse material with fines in Old Tampa Bay is larger than that identified in middle Tampa Bay and the bottom material in Hillsborough Bay is finer than that in middle Tampa Bay, no resuspension or water-quality monitoring sites were established in middle Tampa Bay.

\section{SUMMARY}

Fathometer transects and grab samples were used to determine the size distribution of surficial bed sediment in upper Tampa Bay. The fathometer allows a large area to be covered efficiently, and the grab samples relate the fathometer data to laboratory and field determined sediment size classifications. The resulting sediment size classifications are consistent with findings of previous studies. The finest sediments are in Hillsborough Bay, coarse \$ediments become more prevalent closer to the mouth of Tampa Bay, and the level parts of the bay in relatively deep water have finer bed sediments than shallow-water areas. Based on the sediment size classification data, resuspension monitoring sites were located in large areas of homogeneous bottom material in Old Tampa Bay and in Hillsborough Bay. Shallow-water monitoring sites for comparative water-quality sampling and periodic resuspension monitoring were located in large areas of predominantly coarse, sandy bed materials in these two bays. 


\section{REFERENCES}

Amos, C.L., and Tee, K.T., 1989, Suspended sediment transport processes in Cumberland Basin, Bay of Fundy: Journal of Geophysical Research, v. 94, no. C10, p. 14407-14417.

Boler, Richard, ed., 1987, Water quality, 1984-1985, Hillsborough County, Florida: Hillsborough County Environmental Protection Coumission, $219 \mathrm{p}$.

Bowles, J.E., 1978, Engineering properties of soils and their measurement: New York, McGraw-Hill, 213 p.

Dennison, W.C., 1987, Effects of light on seagrass photosynthesis, growth and depth distribution: Aquatic Botany, v. 27, no. 1, p. $15-26$.

Dolan, D.M., and Bierman, V.J., 1982, Mass balance modeling of heavy metals in Saginaw Bay, Lake Huron: Journal of Great Lakes Research, v. 8, no. 4, p. 676-694.

Doyle, L.J., 1975, Economic geology of shell deposits of Tampa Bay, Florida, United States: Ninth International Congress of Sedimentology, Nice, France, November 23, 1974, theme 9, p. 31-35.

Foth, H.D., 1978, Fundamentals of soil science (6th ed.): New York, John Wiley, $436 \mathrm{p}$.

Goodell, H.G., and Gorsline, D.S., 1961, A sedimentologic study of Tampa Bay, Florida: International Geological Congress, Norden 21 st session, pt. 23, p. 75-88

Goodwin, C.R., 1987, Tidal-flow, circulation, and flushing changes caused by dredge and fill in Tampa Bay, Florida: U.S. Geological Survey Water-Supply Paper 2282, 88 p.

Grant, Jonathan, and Bathmann, U.V., 1987, Swept away: Resuspension of bacterial mats regulates benthic-pelagic exchange of sulfur: Science, v. 236, p. 1472-1474.

Hamblin, P.F., 1989, Observations and model of sediment transport near the turbidity maximum of the upper Saint Lawrence estuary: Journal of Geophysical Research, v. 94, no. C10, p. 14419-14428.

Hamilton, E. L., 1970, Reflection coefficients and bottom losses at normal incidence computed from Pacific sediment properties: Geophysics, v. 35, no. 6, p. 995-1004.

Johansson, J.O.R., and Squires, A.P., 1989, Surface sediments and their relationship to water quality in Hillsborough Bay, a highly impacted subdivision of Tampa Bay: Washington, D.C., National Oceanic and Atmospheric Administration Estuary-of-the-Month Seminar Series no. 11, p. 129-143.
Kirby, R.R., Hobbs, C.H., and Mehta, A.J., 1989, Fine sediment regime of Lake Okeechobee, Florida: University of Florida Coastal and Oceanographic Engineering Department report UFL/COEL-89/009, 77 p.

Lang, G., Schubert, R., Markofsky, M., Fanger, H.U., Grabemann, 1., Krasemann, H.L., Neumann, L.J.R., and Riethmuller, R., 1989, Data interpretation and numerical modeling of the mud and suspended sediment experiment 1985: Journal of Geophysical Research, v. 94, no. C10, p. 14381-14393.

Li, Y.H., Burkhardt, Linda, and Teraoka, Hisayuki, 1984, Desorption and coagulation of trace elements during estuarine mixing: Geochimica et Cosmochemica Acta, v. 48, p. 18791884.

National Oceanic and Atmospheric Administration, 1984, Tampa Bay, northern part (35th ed.): Nautical chart 11413, scale 1:40,000, 1 sheet.

Raytheon Marine Company, [1982], Model DE-719C fathometer precision survey depth recorder instruction manual: Document 1035188.

Ross, F.W., 1975, Sedimentary structures and animal-sediment relationships, Old Tampa Bay, Florida: Tampa, University of South Florida, M.S. thesis, 129 p.

Schoellhamer, D.H., 1990, Observation of sediment resuspension in Old Tampa Bay, Florida: National Conference on Hydraulic Engineering, Proceedings, July 30-August 3, 1990 , San Diego, Calif., v. 1, p. 51-56.

Short, F.T., 1987, Effects of sediment nutrients on seagrasses: Literature review and mesocosm experiment: Aquatic Botany, v. 27, no. 1, p. 41-57.

Smith, D.T., and Li, W.N., 1966, Echo-sounding and sea-floor sediments: Marine Geology, v. 4, p. 353-364.

Stahl, L.E., 1970, The marine geology of Tampa Bay: Tallahassee, Florida State University, M.S. thesis, 70 p.

Taylor, J.L., and Saloman, C.H., 1969, Sediments, oceanographic observations, and floristic data from Tampa Bay, Florida, and adjacent waters, 1961-65: U.S. Fish and Wildlife Service Data Report 34, $561 \mathrm{p}$.

U.S. Department of the lnterior, 1969, Problems and management of water quality in Hillsborough Bay, Florida: Federal Water Pollution Control Administration, $88 \mathrm{p}$.

U.S. Geological Survey, 1989, Water resources data for Florida, water year 1988, southwest Florida surface water: U.S. Geological Survey Water-Data Report FL-88-3A, 290 p.

Willis, J.W., 1984, The shallow structure of Tampa Bay: Tampa, University of South Florida, M.S. thesis, 85 p. 